\title{
MOLECULAR REPLICATOR DYNAMICS
}

\author{
BÄRBEL M. R. STADLER \\ Max Planck Institute for Mathematics in the Sciences \\ Inselstraße 22-26, D-04103 Leipzig, Germany* \\ Institut für Theoretische Chemie und Strukturbiologie, Universität Wien \\ Währingerstraße 17, A-1090 Wien, Austria \\ stadler@mis.mpg.de \\ PETER F. STADLER \\ Lehrstuhl für Bioinformatik, Institut für Informatik, Universität Leipzig, \\ Kreuzstraße 7a, D-04103 Leipzig, Germany \\ Institut für Theoretische Chemie und Strukturbiologie, Universität Wien \\ Währingerstraße 17, A-1090 Wien, Austria \\ The Santa Fe Institute, 1399 Hyde Park Rd., Santa Fe NM 87501 \\ peter.stadler@tbi.univie.ac.at
}

Version of $(28 / \mathrm{Aug} / 2002)$

\begin{abstract}
Template-dependent replication at the molecular level is the basis of reproduction in nature. A detailed understanding of the peculiarities of the chemical reaction kinetics associated with replication processes is therefore an indispensible prerequisite for any understanding of evolution at the molecular level. Networks of interacting self-replicating species can give rise to a wealth of different dynamical phenomena, from competitive exclusion to permanent coexistence, from global stability to multi-stability and chaotic dynamics. Nevertheless, there are some general principles that govern their overall behavior.

We focus on the question to what extent the dynamics of replication can explain the accumulation of genetic information that eventually leads to the emergence of the first cell and hence the origin of life as we know it. A large class of ligation based replication systems, which includes the experimentally available model systems for template directed self-replication, is of particular interest because its dynamics bridges the gap between the survival of a single fittest species to the global coexistence of everthing. In this intermediate regime the selection is weak enough to allow the coexistence of genetically unrelated replicators and strong enough to limit the accumulation of disfunctional mutants.
\end{abstract}

Keywords: self-replication, ligation, autocatalytic network, quasispecies, hypercyle, replicator equation, chemical kinetics, emergence

*Address for correspondence. Tel.: ++49 3419959 518, Fax: ++49 3419959658 


\section{Molecular Replicators}

The notion of a replicator - originally invented by Richard Dawkins [19, pp.1321] — is now used in biology for "an entity that passes on its structure largely intact in successive replications" [138]. The origin of life is characterized by the emergence of heritable information that, through the interplay of selection and variation, leads to Darwinian evolution [58]. The appearance of the first replicator thus marks the transition between the worlds of prebiotic chemistry and primitive biology. The uniformity of the biochemistry in all known living organisms suggests that all modern organisms descend from a last common ancestor, which according to a detailed analysis of protein sequences had a complexity comparable to that of a simple modern bacterium and lived some 3.2-3.8Gyr ago.

The formation of the Solar System began about 4.6Gyr ago in the aftermath of a local supernova explosion. The inner planets were probably formed by collision of some 500 Moon-sized planetesimals [140]. It is unlikely that Earth could have sustained life before about 4.2Gyr or even 4.0 Gyr because of meteorite bombardment [108]. Depending on the details of the prebiotic environment a large variety of organic compounds may have been available about 4.0 Gyr ago, including amino acids, hydroxy acids, sugars, purines, pyrimidines and fatty acids $[68,12,147]$. Geological evidence [78], on the other hand, shows that it is certain that life has been present on Earth for at least 3.5Gyr, and it is probable that life began before 3.8Gyr $[93,89]$.

There are strong reasons to conclude that the Last Common Ancestor was preceded by simpler life forms that were based primarily on RNA. This era, during which the genetic information resided in the sequence of RNA molecules and the phenotype derived from the catalytic properties of RNA, has been termed the $R N A$ world $[37,36]$. In this scenario, the translation of RNA into proteins and, finally, the usage of DNA [35] as information storage device are later inventions. A rather detailed model of the steps leading from the RNA world to modern cellular architectures is discussed in [84]. The RNA world scenario is supported both by the wide range of catalytic activities that can be realized by relatively small ribozymes [52,57,58,64,131], Fig. 1, and by the usage of RNA catalysis at crucial points in modern cells $[56,22,76]$. Plausible ribozyme catalyzed pathways for a late-stage ribo-organism are discussed in [58], the role and evolution of co-enzymes in the RNA world is explored in [53].

The ribozymes in Fig. 1, in particular the replicase (a), are probably still too large to arise spontaneously; it seems much more plausible that molecules such as these arose from smaller, simpler molecules with less sophisticated functionalities.

Julius Rebek and others $[79,129,29]$ have demonstrated that autocatalytic chemical reaction systems can be assembled in which organic molecules undergo selfreplication. Autocatalysis in such systems of small molecules is certainly interesting because it reveals some mechanistic details of molecular recognition. However, these systems are hardly at the basis of biological replication since they cannot store a siz- 


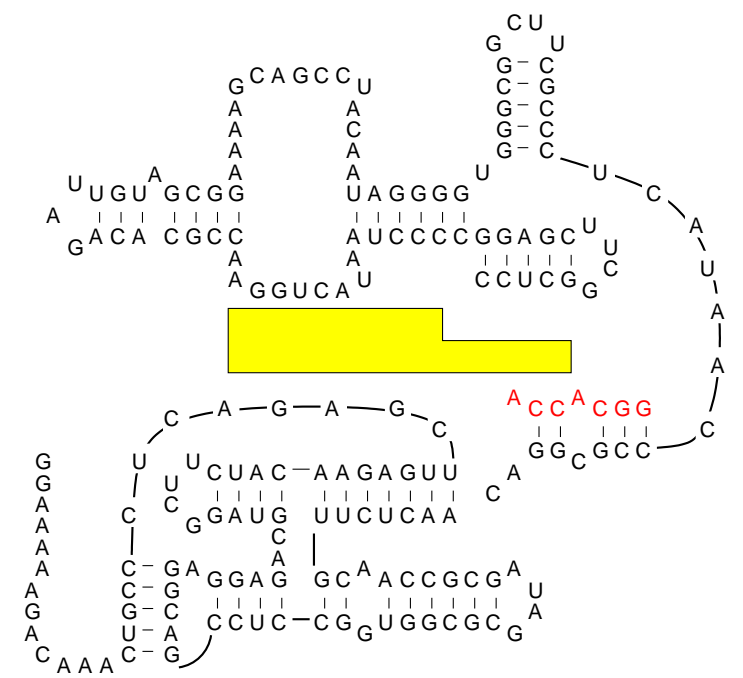

(a)

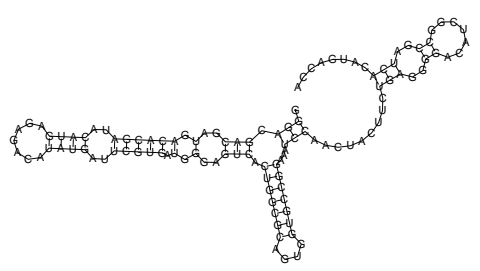

(c)

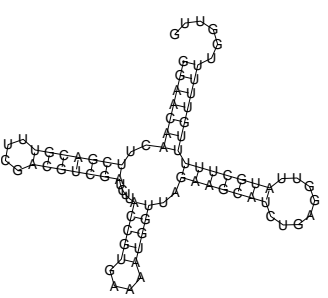

(d)

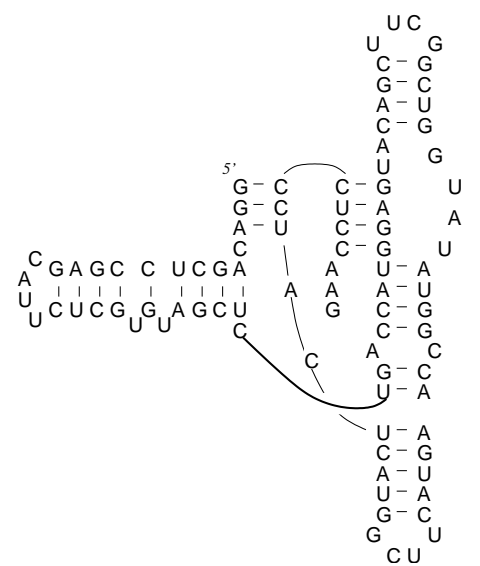

(b)

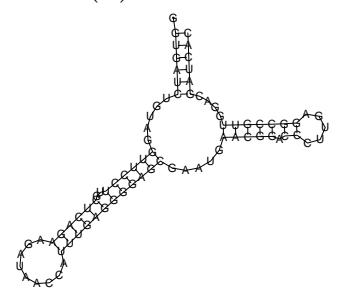

(e)

Fig. 1. Examples of artificial ribozymes with catalytic functions that are interesting in the context of the RNA world. (a) RNA replicase 18 [57] (b) 16.min ligates RNA to protein [7] (c) A ribozyme that activates amino acids [61] (d) ATrib [64] can self-aminoacylate and (e) a ribozyme that catalyzes tRNA aminoacylation [64].

able amount of information. Consequently, there is no room for molecular variants, "mutations", and hence these systems cannot sustain Darwinian evolution.

The simplest way of overcoming this limitation is to envision some kind of polymer as the carrier of genetic information, just as DNA takes on this role in present day cells. RNA has the same capacity, which is actually made use of by a large class of viruses. Polypeptides are the obvious alternative candidates.

Template-induced synthesis of longer RNA molecules from monomers in the absence of an enzyme, however, has not been successfully achieved so far [82]. Autocatalytic template-induced synthesis of oligonucleotides from smaller oligonucleotide precursors, however, was successful: a hexanucleotide through ligation of two trideoxynucleotide precursors was carried out by Günter von Kiedrowski [135], see also $[137,106]$. More recently it was shown that a peptide comprised of 32 amino acids can undergo exactly the same type of autocatalytic synthesis from two roughly equal fragments [62], Fig. 2. A recent experimental study using nucleic acids [54] 

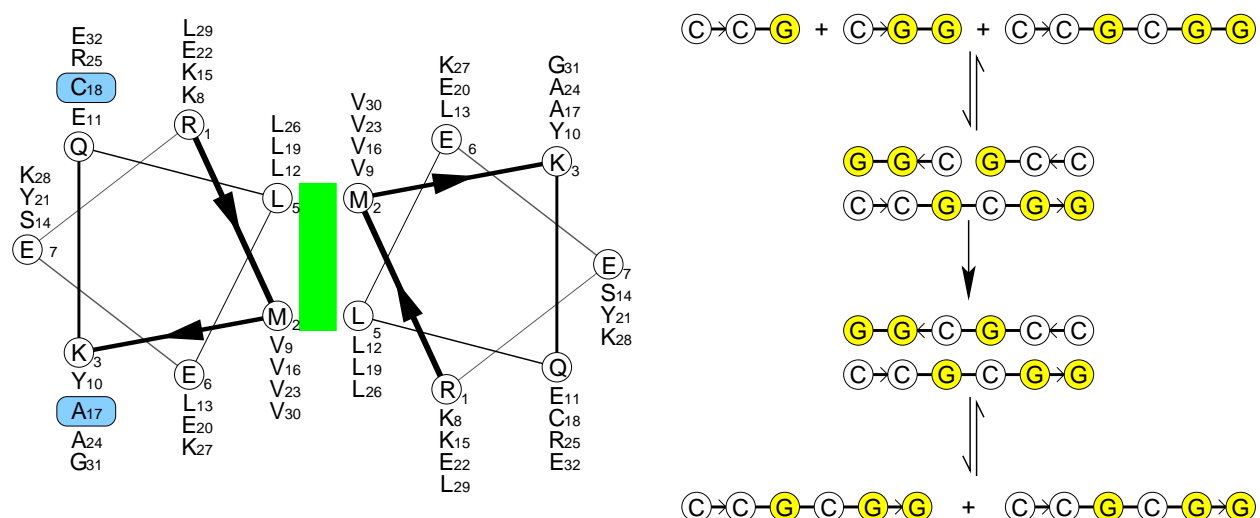

Fig. 2. Reza Ghadiri's [63] self-replicating peptide $\mathfrak{T}$ acts as a template for ligation of the two fragments $\mathfrak{E}$ and $\mathfrak{N}$, where

$$
\begin{aligned}
& \mathfrak{E}=\mathrm{Ar} \cdot \mathrm{RMKQLEEKVYELLSKVA} \cdot \mathrm{CO} \cdot \mathrm{S} \cdot \text { Benzyl } \\
& \mathfrak{N}=\mathrm{H}_{2} \mathrm{~N} \cdot \mathrm{CLEYEVARKJJKGE} \cdot \mathrm{CO} \cdot \mathrm{NH}_{2} \\
& \mathfrak{T}=\mathrm{RMKQLEEKVYELLSKVA}: \text { CLEYEVARKJJKGE }
\end{aligned}
$$

and $\mathrm{Ar}=4$-acetamidobenzoyl. The ligation site is located between $A_{17}$ and $C_{18}$. Günter von Kiedrowski's [135] self-replicating palindromic RNA reproduces by means of ligation of the two trinucleotides.

emphasizes the importance of ligation-based replication mechanisms for the origin of life.

An interesting feature of oligopeptide self-replication concerns easy formation of higher replication complexes: Coiled-coil formation is not restricted to two interacting helices, triple helices and higher complexes are known to be very stable too. Autocatalytic oligopeptide formation may thus involve not only a template and two substrates but, for example, a template and a catalyst that form a triple helix together with the substrates $[63,145]$. We will return to such higher order replication systems in section 6 .

A major shortcoming of peptide replication, however, is the fact that only a very small fraction of all possible sequences fold into three-dimensional structures that are suitable for leucine zipper formation and hence a given autocatalytic oligopeptide is very unlikely to retain the capability of template action on mutation. The (rather large) leucine zipper sequences of the building block must themselves be somehow instructed somewhere in the system. Peptides are therefore only occasional templates; their evolutionary adaptability is crippled by the fact that mutants are unlikely to be templates themselves. In contrast to the volume filling principle of protein packing, specificity of catalytic RNAs is provided by base pairing and to a lesser extent by tertiary interactions. Both are the results of hydrogen bond specificity. Template action of nucleic acid molecules, being the basis for replication, results directly from the structure of the double helix. It requires an appropriate backbone provided by the anti-parallel ribose-phosphate or 2'-deoxyribosephosphate chains and a suitable geometry of the complementary purine-pyrimidine 

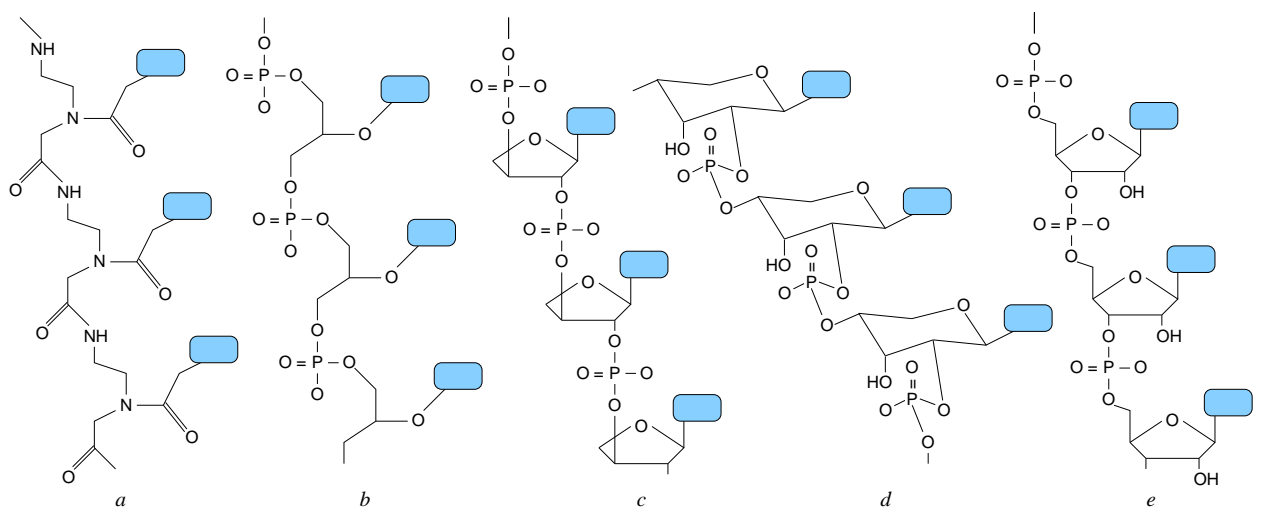

Fig. 3. Alternatives to RNA. A pre-RNA world may have existed where genetic information was stored in alternative pre-bio-polymers. (a) PNA (peptide nucleic acid) [77], (b) glycerol-derived nucleic-acid analogue [17], (c) TNA (threose nucleic acid) [92], (d) pyranosyl-RNA [83], (e) RNA for comparison.

pairs. All RNA (and DNA) molecules, however, share these features which, accordingly, are independent of sequence. Every RNA molecule has a uniquely defined complement. Nucleic acid molecules, in contrast to proteins, are therefore obligatory templates. This implies that mutations are conserved and readily propagated into future generations. A somewhat different classification of replicators in terms of their combinatorial limitations can be found in [124].

While some classes of non-instructed polymers, such as random oligopeptides of the proteinoid type [32], are plausible products of prebiotic chemistry, this is not the case for RNA $[60,100,105]$. Heritable molecular information therefore most likely has its origins in a different chemical system. Two widely different scenarios have been proposed: One possibility is that nucleic acid like polymers with different backbones and maybe different side chains predated the RNA world. There is no shortage of plausible candidates, including tetrose based nucleic acids and peptide nucleic acids, Fig. 3.

Another alternative is the container first hypothesis that assumes the existence of autocatalytically replicating lipid micelles or vesicles, a possibility that was demonstrated experimentally by Luisi and coworkers $[6,139,69,133]$. Doron Lancet and Daniel Segré suggest that such "proto-cells" might have a complex lipid composition that is faithfully transferred when the vesicle duplicates, see e.g. $[103,104,102]$. Of course, a sizable amount of information can be accumulated only if a very large set of chemically distinct lipids can be brought together in a single vesicle. In this Lipid World scenario, the catalytic activities of the complex lipid phase would eventually instruct the synthesis of peptides and nucleic acids.

For the purpose of this article, fortunately, the precise chemical instantiation of the earliest replicons is largely irrelevant, because their basic kinetic properties depend more on the logics of the replication mechanism than on the molecular 
details. In this contribution we shall focus on the consequences of the peculiar chemical reaction kinetics that is implied by replication. We will consider two times two classes of models in some detail: template induced replication with and without an additional self-replicating catalyst, both with simple mass action kinetics and with a chemically more realistic ligation-like mechanism. The common theme of these models is the emergence and persistence of a sufficient amount of genetic information for biological evolution to take off.

\section{The Molecular Quasispecies and "Survival of the Fittest"}

Let us begin with the simplest possible replication scheme

$$
\mathrm{A}+\mathrm{C} \stackrel{\mathrm{E}}{\longrightarrow} 2 \mathrm{C}+\mathrm{A}^{\prime}
$$

in which some energy rich building material $A$ is used to produce copies of the template $\mathrm{C}$. This describes the logic, but not necessarily the details of the reaction kinetics, of the examples in Fig. 2.

The first successful attempts to study RNA evolution in vitro were carried out in the late sixties by Sol Spiegelman and his group [75,109]. They created a "protein assisted RNA replication medium" by adding an RNA replicase isolated from $E$. coli cells infected by the RNA bacteriophage $\mathrm{Q} \beta$ to a medium for replication that also contained the four ribonucleoside triphosphates in a suitable buffer solution. $\mathrm{Q} \beta$ RNA and some of its smaller variants start instantaneously to replicate when transferred into this medium.

Extensive studies on the reaction kinetics of RNA replication in the $\mathrm{Q} \beta$ replication assay were performed by Christof Biebricher in Göttingen [8]. These studies revealed consistency of the kinetic data with a multi-step reaction mechanism. Depending on concentration the growth of template molecules allows to distinguish three phases of the replication process: (i) at low concentration all free template molecules are instantaneously bound by the replicase which is present in excess and therefore the template concentration grows exponentially, (ii) excess of template molecules leads to saturation of enzyme molecules, then the rate of RNA synthesis becomes constant and the concentration of the template grows linearly, and (iii) very high template concentrations impede dissociation of the complexes between template and replicase, and the template concentration approaches a constant in the sense of product inhibition. We neglect plus-minus complementarity in replication by assuming stationarity in relative concentrations of plus and minus strand [25] and consider the plus-minus ensemble as a single species. Then, RNA replication may be described by the over-all mechanism:

$$
\mathrm{A}+\mathrm{C}_{\mathrm{i}}+\mathrm{E} \underset{\bar{k}_{i}}{\stackrel{k_{i}}{\rightleftharpoons}} \mathrm{A}+\mathrm{C}_{\mathrm{i}} \cdot \mathrm{E} \stackrel{a_{i}}{\longrightarrow} \mathrm{C}_{\mathrm{i}} \cdot \mathrm{E} \cdot \mathrm{C}_{\mathrm{i}} \underset{\bar{k}_{i}^{\prime}}{\stackrel{k_{i}^{\prime}}{\rightleftharpoons}} \mathrm{C}_{\mathrm{i}} \cdot \mathrm{E}+\mathrm{C}_{\mathrm{i}}
$$

This simplified reaction scheme reproduces all three characteristic phases of the detailed mechanism and can be readily extended to replication and mutation. 
Evolution of molecules based on replication and mutation exposed to selection at constant population size has been formulated and analyzed in terms of chemical reaction kinetics $[25,73,26,27]$. Error-free replication and mutation are parallel chemical reactions,

$$
\mathrm{A}+\mathrm{C}_{\mathrm{i}} \stackrel{Q_{j i} a_{i}}{\longrightarrow} \mathrm{C}_{\mathrm{j}}+\mathrm{C}_{\mathrm{i}}
$$

and form a network which in principle allows to form every RNA genotype as a mutant of any other genotype. The material A required for or consumed by RNA synthesis is continuously replenished. The quantities of interest are the relative concentrations $x_{k}=\left[C_{\mathrm{k}}\right] / c_{0}$ where $c_{0}=\sum_{k}\left[C_{\mathrm{k}}\right]$ is the total concentration of replicating material. The reaction scheme 3 translates into the kinetic equations

$$
\dot{x}_{k}=x_{k}\left(a_{k} Q_{k k}-\Phi(t)\right)+\sum_{j \neq k} Q_{k j} a_{j} x_{j} .
$$

The diagonal elements of $Q$ are the replication accuracies, i.e., the fractions of correct replicas produced on the corresponding templates. The time dependent excess productivity which is compensated by the flow in the reactor is the mean value $\Phi(t)=\sum a_{j} x_{j}$. The selective value of $C_{k}$ is the diagonal element $w_{k k}$ of matrix $W$ with the entries $w_{k j}=a_{k} Q_{k j}$.

The selective value of a genotype is tantamount to its fitness in the case of vanishing mutational back-flow and hence the genotype $C_{m}$ with maximal selective value, $w_{m m}=\max _{j} w_{j j}$, dominates a population after it has reached the selection equilibrium. It is called the master sequence. The notion of a quasispecies was introduced for the stationary genotype distribution in order to point at its role as the genetic reservoir of the population. In the simplest case one considers only point mutations. This leads to the explicit expression

$$
Q_{k j}=(1-p)^{n}\left(\frac{p}{1-p}\right)^{d_{k j}}
$$

where $d_{k j}$ is the Hamming distance of the two sequences $C_{k}$ and $C_{\mathbf{j}}$ and $p$ is the per-digit error rate.

The stationary frequencies $x_{k}$ can be computed explicitly in many cases, e.g. when the master sequence is derived from the single peak model landscape that assigns a higher replication rate to the master and identical values to all others $[121,125,1]$. The master sequence vanishes at some finite replication accuracy that depends on the superiority and the length $n$ of the replicating sequences. The critical value $p_{\max }$ of the mutation rate is known as the error threshold. Above the threshold no stationary distribution of sequences is formed. Instead, the population drifts randomly through sequence space. This implies that all genotypes have only finite life times, inheritance breaks down, and evolution becomes impossible. The effects of finite population sizes on the error threshold are considered e.g. in $[80,2,15]$.

Variations in the accuracy of in vitro replication can indeed be easily achieved because error rates can be tuned over many orders of magnitude [65,67]. The range 


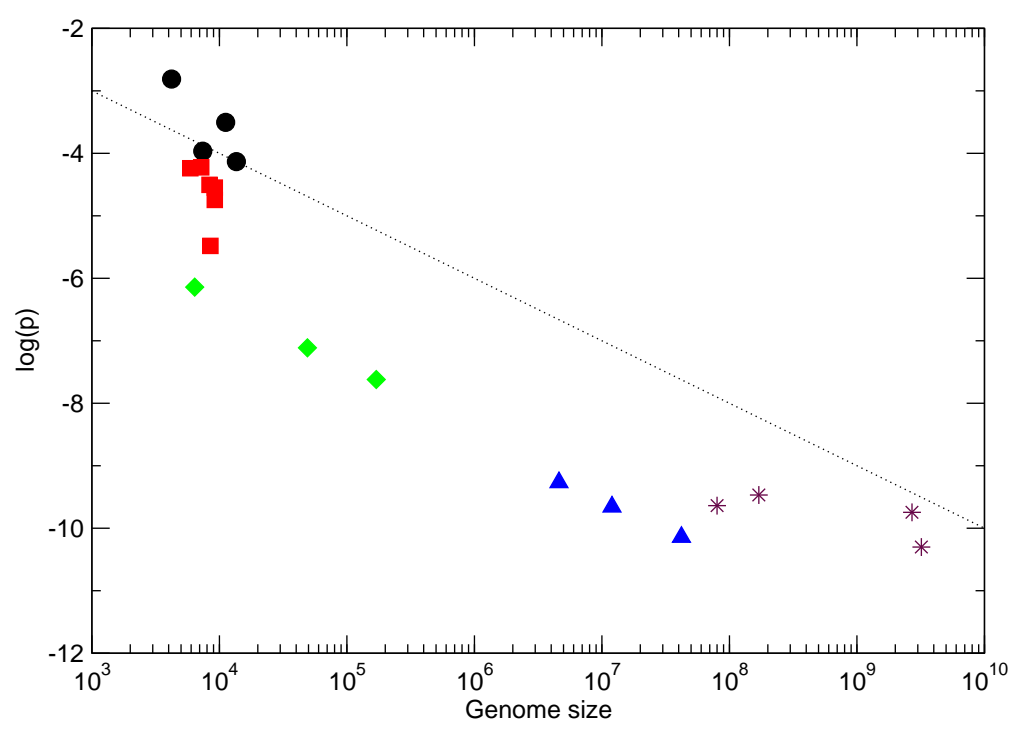

Fig. 4. Relationship between genome length and mutation rate. $\bullet$ single stranded RNA viruses, retro-transcribing viruses and transposons, DNA viruses, $\boldsymbol{\Delta}$ Bacteria, * Eukarya. Data are taken from [24]. The dotted line is $p=1 / n$.

of replication accuracies that are suitable for evolution is limited, however, by the maximal accuracy that can be achieved by the replication machinery and the minimum accuracy determined by the error threshold. Populations in constant environments have an advantage when they operate near the maximal accuracy because then they loose as few copies through mutation as possible. In highly variable environments the opposite is true: it pays to produce as many mutants as possible because then the chance is largest to cope successfully with change.

In order to check the relevance of the error threshold for the replication of RNA viruses the minimum accuracy of replication can be transformed into a maximum chain length $n_{\max }$ for a given error rate $p$. The condition for stationarity of the quasispecies reads

$$
n<n_{\max }=-\frac{\ln \sigma}{\ln (1-p)} \approx \frac{\ln \sigma}{p}
$$

where $\sigma$ is the superiority of the master species, see e.g. [27]. The populations of most RNA viruses were indeed shown to live near the critical value of replication accuracy $[20,21]$. Moreover, the genome size $n$ of any organism is roughly the inverse mutation rate per site and replication [23,24], see Fig. 4.

Equ.(6) imposes a fundamental limitation on the size of the self-replicating biopolymers. In the following sections we shall encounter mechanisms by which this limitation can be circumvented. 


\section{Replicator Equations}

So far we have neglected any direct interactions between the replicating species. In the most general case we have

$$
C_{k} \stackrel{C_{1}, C_{2}, \ldots, C_{n}}{\longrightarrow} 2 C_{k},
$$

where the replication rate $f_{k}$ of $C_{k}$ is influenced by the concentrations of all the species $C_{1}$ through $C_{n}$. Most papers on autocatalytic reaction networks (including much of the work of the group in Vienna) use the replicator equation $[96,47,48]$

$$
\dot{x}_{k}=x_{k}\left[f_{k}(x)-\sum_{j=1}^{n} x_{j} f_{j}(x)\right]
$$

despite the fact that a continuously stirred tank reactor (CSTR) would in many cases be a more appropriate description of the biological or chemical situation. This simplification is motivated by the equivalence of the two models for the special case of homogeneous interaction functions [97]. Furthermore, it can be shown that the CSTR dynamics converges to the replicator dynamics in the limit of small flux rates [40]. We remark that the quasispecies model yields, in the limit of vanishing mutation rates, a replicator equation with constant growth functions $f_{k}(x)=a_{k}$.

Let us now return to the case of interacting replicators. The simplest example assumes reactions that are catalyzed by a single replicator, i.e.,

$$
\mathrm{C}_{\mathrm{k}}+\mathrm{C}_{\mathrm{j}} \longrightarrow 2 \mathrm{C}_{\mathrm{k}}+\mathrm{C}_{\mathrm{j}}
$$

Assuming, furthermore, that these reaction follow mass action kinetics, the growth functions $f_{k}$ in equ.(8) are linear,

$$
f_{k}(x)=\sum_{j=1}^{n} a_{k j} x_{j}
$$

with $a_{k j} \geq 0$. These 2nd-order replicator equations also describe the dynamics of strategies in evolutionary games [126]. Hofbauer [42] showed that they are topologically equivalent to the Lotka-Volterra equations

$$
\dot{y}_{k}=y_{k}\left(r_{k}+\sum_{j=1}^{n-1} b_{k j} y_{j}\right)
$$

which are a standard model in mathematical ecology. Indeed, recent experimental research in the group of John McCaskill $[142,74]$ is dealing with molecular ecologies of strongly interacting molecular replicators.

A particularly important class of 2 nd order replicator equations are the catalytic networks [99] characterized by $a_{k j} \geq a_{j j}$, i.e, the networks in which the catalytic assistance for all other replicators is at least as strong as self-catalysis. The interaction structure of catalytic networks is conveniently represented as a directed 

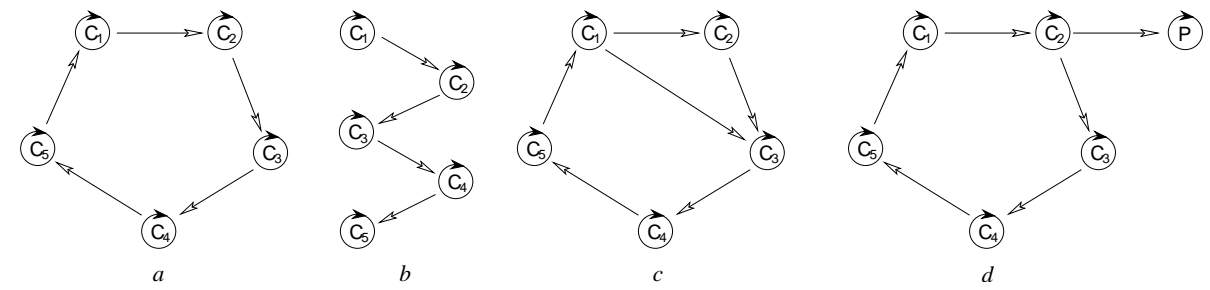

Fig. 5. Architectures of catalytic networks. (a) Hypercycle, (b) catalytic chain, (c) hypercycle with "short-cut", (d) hypercycle with a parasite.

graph $\Gamma(\mathbf{A})$ in which the vertices are the replicating species and there is an edge $\left[\mathrm{C}_{\mathrm{j}}\right] \rightarrow\left[\mathrm{C}_{\mathrm{k}}\right]$ if $a_{k j}-a_{j j}>0$, i.e., if $\left[\mathrm{C}_{\mathrm{j}}\right]$ advances the replication of $\left[\mathrm{C}_{\mathrm{k}}\right]$, Fig. 5.

The dynamics of a catalytic network is at least in part determined by its graph structure. For example, coexistence is impossible in linear chains, Fig. 5b. The hypercycle, on the other hand, was introduced as a possibility to overcome the limitations of the error threshold [28]. The positive feedback cycle, in which each template promotes the replication of the next one guarantees the permanent coexistence of all its members. While hypercycles are stable against dynamical perturbations, they are unstable against structural perturbations of the networks. Both "short cuts", Fig. 5c, and parasites, Fig. 5d, may lead to dynamically unstable networks.

An important concept in the theory of replicator equations is that of a saturated fixed point [44]. Let $F_{K}$ be the face of the concentration simplex $S_{n}$ whose (relative) interior is defined by $x_{k}=0$ for all species $k \in K$. An eigenvector of (the Jacobian matrix of) the vector field at a point $x^{K} \in F_{K}$ that points out of this plane is a called a transversal eigenvalue. We have $\lambda_{k}\left(x^{K}\right)=f_{k}\left(x^{K}\right)-\Phi$ for $k \in K$ and $x^{K} \in F_{K}$. A fixed point $\hat{x} \in F_{K}$ is saturated if none of its transveral eigenvalues is positive.

So far, we have excluded mutation from our considerations. Mutation can be treated as a perturbation at least in the limit of small mutation rates [118]. Since mutation by construction points inward on the boundary of the simplex, one obtains the rest point migration theorem: If a fixed point is saturated it "moves" into the interior of the simplex when mutation is switched on; all other equilibria are pushed into the non-physical exterior. Mutation thus may simplify the phase portraits considerably. Larger mutation rates, however, may lead to complicated bifurcation patterns, an increase in the number of competing equilibria, and even the appearance of limit cycles $[43,116]$.

The dynamics of second order replicator equations can be extremely complicated despite the rather simple form of the differential equation. In the case of two independent variables ( $n=3$, the state space is an equilateral triangle) there are 35 different generic phase portraits [10,117]. It is interesting to note that this rather involved classification remains essentially unchanged under monotonous transformations of the linear growth functions, i.e., for $f_{k}(x)=\vartheta\left([\mathbf{A} x]_{k}\right)$, see [111]. The 

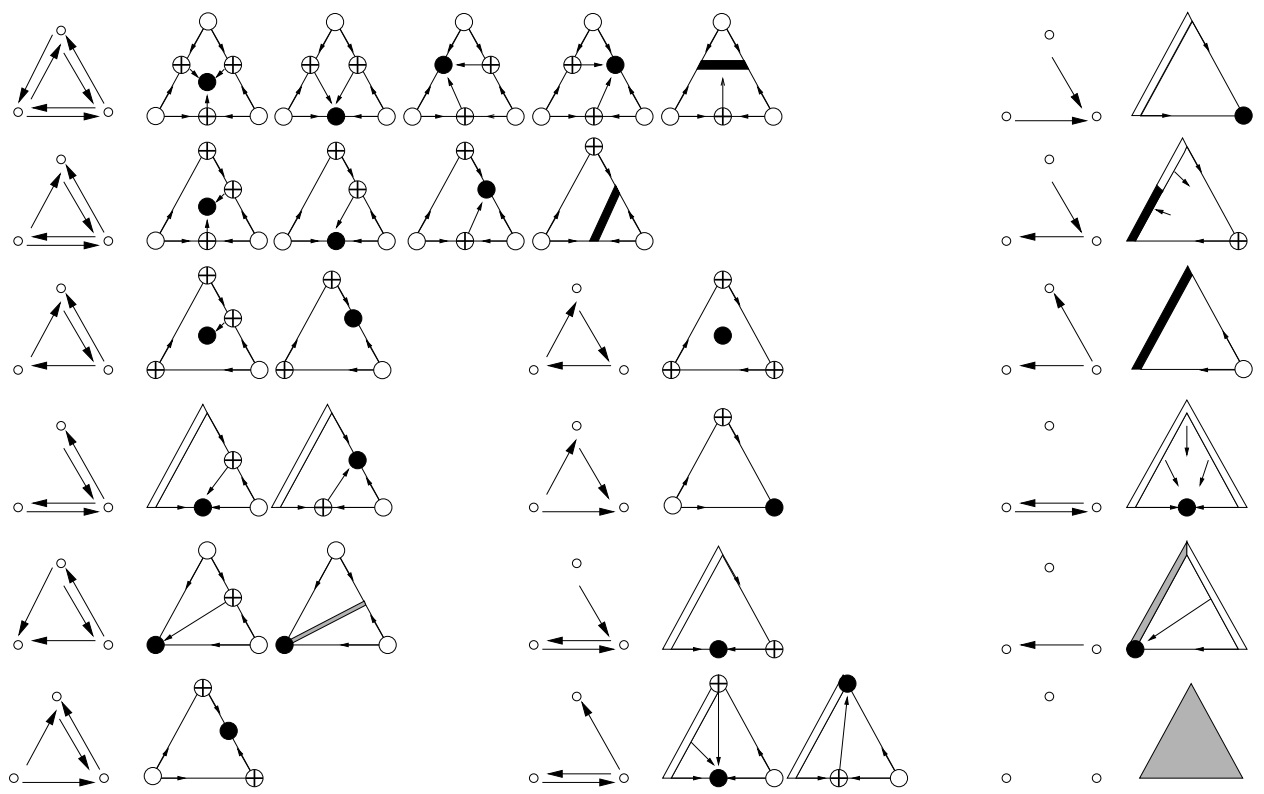

Fig. 6. Classification of autocatalytic networks with $n=3$ species. The interactions are represented as graphs with a directed edge iff $a_{k j}-a_{j j}>0$.

classification of three-species catalytic networks is shown in Fig. 6. In the case of three independent variables, i.e. $n=4$ species, there are heteroclinic orbits, multiple limit cycles, and strange attractors $[13,49,38,132,5,91]$, Fig. 7 .

Often one is not interested in all details of a dynamical system or in the structure of its $\omega$-limit sets, less detailed knowledge may well be sufficient. Probably the most important question is: Can all species coexist in the system for arbitrarily long time? Or will some species die out in the long run? Schuster et al. [98] introduced the notion of permanence (permanent coexistence) to formalize this question. A variety of different notions of cooperation, the first of which is now called weak persistence [34], have been proposed by various authors, e.g. [33,51].

A replicator equation (8) is said to be permanent if there is a compact subset $C$ of the interior of the state space $S_{n}$, such that any trajectory starting in the interior of $S_{n}$ will eventually end up in $C$. In other words, there is a (possibly very thin but finite) repulsive "skin" on the boundary of the state space.

A sufficient (but for $n \geq 5$ not necessary) criterion for permanence of second order replicator equations was derived by Jansen [55]:

Theorem 3.1. If there is a vector $p \in$ int $S_{n}$ such that for every isolated rest point $x^{K} \in F_{K}$ on the boundary of the simplex holds $\sum_{k \in K} p_{k} \cdot \lambda_{k}\left(x^{K}\right)>0$ then the second order replicator equation is permanent. 

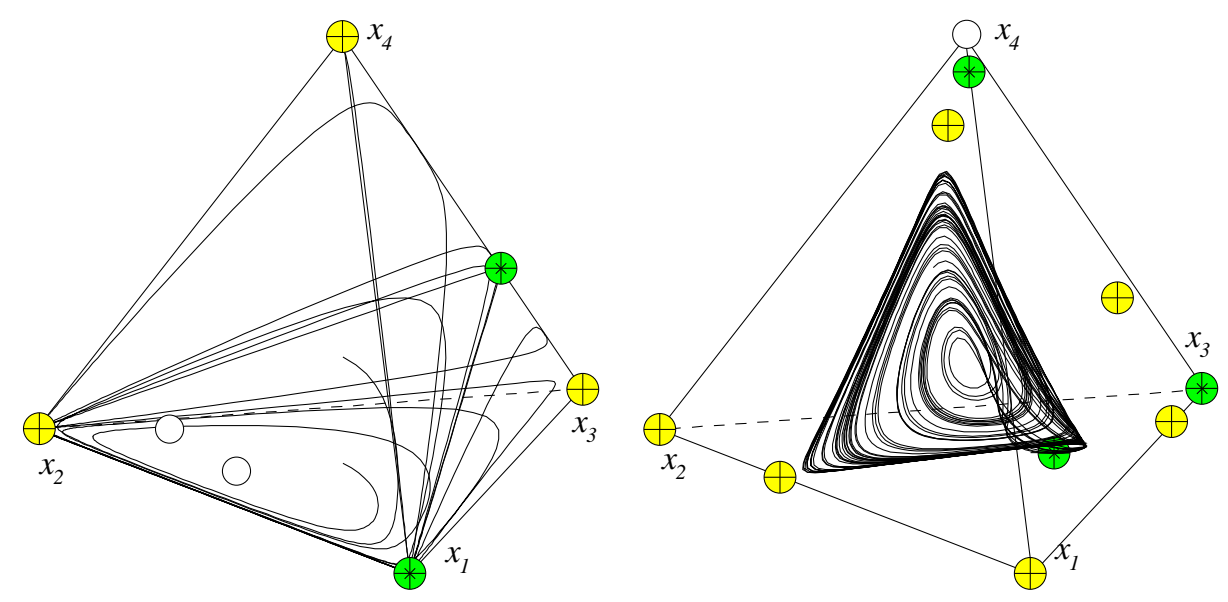

Fig. 7. Complex dynamics in 2nd order replicator equations. Left: an attracting heteroclinic orbit in the central plane [110]. Right: A chaotic attractor of class described in [5]. The interaction matrices are

$$
\mathbf{A}=\left(\begin{array}{cccc}
0 & -2 & 7 & 8 \\
20 & 0 & -7 & -6 \\
-20 & 1 & 0 & 2 \\
-20 & 1 & 1 & 0
\end{array}\right) \quad \mathbf{A}=\left(\begin{array}{cccc}
0 & 0.5 & -0.1 & 0.1 \\
1 & 0 & -0.5 & 0 \\
-0.5 & 1.0 & 0 & 0 \\
1.5 & -0.8 & -0.2 & 0
\end{array}\right)
$$

Fixed points are distinguished by the number of stable directions: sources without stable direction $\circ$, one stable direction $\oplus$, and $\circledast$ two stable directions.

This condition is equivalent to a linear program and hence computationally accessible [55].

In some cases the graph structure of the catalytic network allows conclusions about permanence. For example: If the catalytic network is permanent, then its graph $\Gamma(\mathbf{A})$ is strongly connected [107]. In particular, two hypercycles cannot coexist [45]. For $n \leq 5$ the graph of a permanent catalytic network must contains a Hamiltonian circuit, i.e., a closed path visiting every vertex exactly once [3,47]. Conversely, if $\Gamma$ contains a Hamiltonian circuit then there is a non-negative matrix A with $\Gamma(\mathbf{A})=\Gamma$ such that the second order replicator equation is permanent [119].

\section{The Evolution of Coexistence}

A fundamental necessary condition for any kind of coexistence is posed by the Exclusion Principle [50]:

Theorem 4.1. If a second order replicator network does not have an interior fixed point, then all orbits converge to the boundary of the state space.

Since the existence of a positive solution of $\mathbf{A} x=\Phi \overrightarrow{1}$ is a rather restrictive condition, we have to interpret the Exclusion Principle as a rather pessimistic statement about the prospects of coexistence. Permanence requires an even more restrictive condition: If the system is permanent it has no saturated fixed point on the bound- 


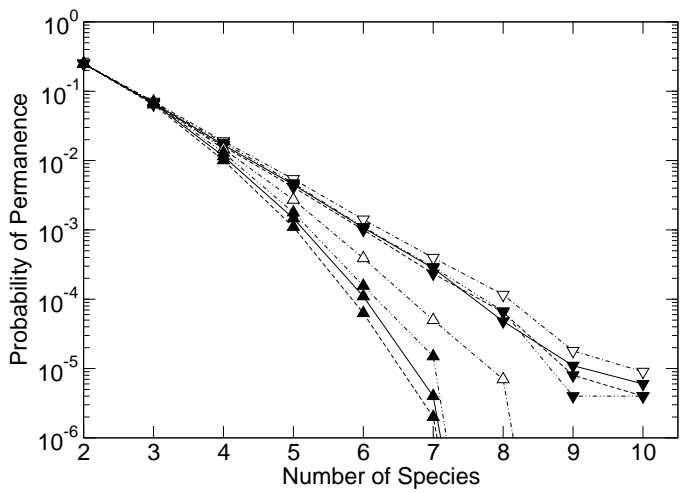

(a)

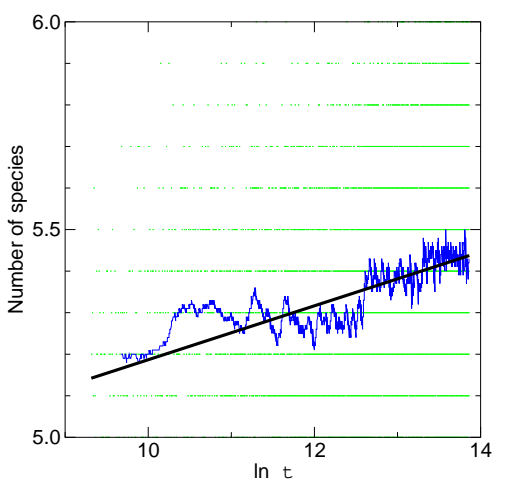

(b)

Fig. 8. (a) Probability of generating a permanent replicator network by chance. Upper bounds are from algebraic conditions on the interaction matrix $\mathbf{A}$, the lower bound is obtained by checking Jansen's criterion (Theorem 3.1). Data are for Gaussian, uniform, Laplace, and Cauchy distributions for the coefficients $a_{i j}^{\prime}=a_{i j}-a_{j j}$. Long tails as in the Cauchy distribution seem to favor permanence [114].

(b) The average number of species increases very slowly by incorporation of mutants. The resulting networks are permanent, highly connected, and (at least approximately) catalytic networks [39].

ary $[44,46]$.

A numerical survey [114] for small and moderate size replicator networks therefore shows that permanence - and cooperativity in general - is a very rare phenomenon, except for very small networks with $n=2,3$, or 4 members. The probability of finding cooperative behavior in random autocatalytic networks of replicator or Lotka-Volterra type decreases at least as $4^{-n}$ with the number of species involved; maybe even faster than exponential, Fig. 8a. Permanence is more likely in catalytic networks and decreases dramatically with the number of inhibitory interactions $a_{k j}-a_{j j}<0$. Even in catalytic networks, however, the probability decreases exponentially.

In the limit of small mutation rates one can model the long time evolution of replicator networks. To this end the differential equation is numerically integrated until the $\omega$-limit is reached. In some cases the appropriate fixed points can also be obtained analytically. Then the interaction matrix $\mathbf{A}$ is modified by (i) removing all rows and columns that correspond to extinct species $x_{k}=0$ and (ii) by adding an additional row and column. In the second step either randomly chosen entries are used to produce a new species that is unrelated to the existing network (an invader) or a row and corresponding column is copied and a small amount of noise is added.

May and Nowak [70] analyzed the evolution of a simple Lotka-Volterra type model of super-infection in host-parasite associations. Interestingly, the same type of equations was obtained in a study on competition and biodiversity in spatially structured habitats [128]. The total number of species in their system slowly in- 
creases, $n(\tau) \propto \ln \tau$, where $\tau$ is the number of mutation events. More recently, a square root law $n(\tau) \sim \sqrt{\tau}$ was found in a co-infection model that neglects competition of different parasites within the same host [71]. For general replicator networks we found that mutation leads to a slow increase of diversity consistent with a logarithmic increase of the number of species with the number of mutation events [39], Fig. 8b, while unrelated invaders repeatedly lead to a complete collapse of the network and hence preclude any long-term growth. The evolved networks have a specific structure in the mutation case: all strong interactions are positive, the interaction matrix is nearly symmetric, and the connectivity is very high.

The mutation mechanism employed in these simulations might not be entirely realistic, however. It seems entirely plausible that some mutants completely loose their catalytic activities while retaining their template properties. Such mutants are parasites $[72,14]$.

Despite their importance for the theory of prebiotic evolution there does not seem to be any systematic treatment of parasitic interactions in replicator networks. A classification of types of parasites in terms of the structure of the networks is also missing; a distinction between "short-cuts" and "true parasites" appears to be useful, see Fig. 5. True parasites might be defined as dead ends (sinks) in the network graph $\Gamma(\mathbf{A})$, i.e., they do not contribute anything to the self-maintenance of the network. In the most general case one might want to consider a species $P$ that dominates another network species $C_{k}$ in the following sense

$$
\mathrm{P} \succ \mathrm{C}_{\mathrm{k}} \quad \Longleftrightarrow \quad f_{\mathrm{P}}(x) \geq f_{k}(x) \text { and } f_{\mathrm{P}}(x)>f_{k}(x) \text { whenever } f_{k}(x)>0 .
$$

Equ.(8) satisfies the "quotient rule"

$$
\frac{\mathrm{d}}{\mathrm{d} t} \ln \frac{x_{k}}{x_{j}}=f_{k}(x)-f_{j}(x)
$$

from which one can deduce that a dominated species will always die out. The argument proceeds by integrating the quotient rule over time and observing that $\ln \frac{x_{k}}{x_{j}}$ must converge to either $+\infty$, or $-\infty$ unless both $f_{k}(x)$ and $f_{j}(x)$ converge to 0 . A parasitic mutant that dominates its ancestor will therefore cause the extinction of the ancestor. In the example of the hypercycle with a parasite, Fig. $5 \mathrm{~d}$, this changes the network graph to a catalytic chain, Fig. 5b; hence all other hypercycle members will also die out. Since the parasite at the end of the chain cannot catalyze its own replication, it will finally disappear as well.

So far we have not considered the detrimental effects of mutation itself on catalyzed replication. Simplified models equations use a single dynamical variable to describe the "error tail" that consisting of all "lethal" mutants $[4,81,115,16]$. Beyond a critical mutation rate this error tail dominates. Christian Forst [31] shows that there is an error threshold also for permanent catalytic networks also without this simplification. Hence genome size is limited by replication accuracy also in the case of cooperating replicators. Once the mutation rate is small enough to sustain an RNA molecule with replicase activity below the error threshold, however, selection 
can act to improve the copying fidelity. This allows for an increased sequence length and thus opens up the possibility for a further decrease of the per base mutation rate [87].

\section{Coexistences Through Product Inhibition}

Eörs Szathmáry [123] pointed out that a simple modification of the replicator equation to a "parabolic" growth law

$$
\dot{x}_{k}=b_{k} x_{k}^{a}-x_{k} \Phi
$$

with $0<a<1$ leads to unconditional coexistence. We remark that a variant of equ.(14) with an explicit decay term $-d_{k} x_{k}$ yields the same qualitative behavior [88]. The case $a=1 / 2$ is obtained as a limiting case of von Kiedrowski's "Minimal Replicator Theory" [136]. Equ.(14) is, however, not physically meaningful on the boundary of the simplex where the Jacobian of the vector field diverges. In [136, 141,113] chemically realistic mechanisms are considered that produce equ.(14) as a limiting case. The full ligation mechanism of both von Kiedrowski's and Ghadiri's examples, Fig. 2 in the introduction, consists of the following steps:

$$
\begin{aligned}
& \mathrm{A}+\mathrm{C} \underset{\bar{a}}{\stackrel{a}{\rightleftharpoons}} \mathrm{AC} \quad \mathrm{AC}+\mathrm{B} \underset{\bar{h}}{\stackrel{h}{\rightleftharpoons}} \mathrm{ABC} \quad \mathrm{ABC} \stackrel{r}{\longrightarrow} \mathrm{C}_{2}
\end{aligned}
$$

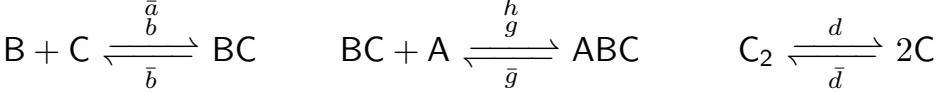

Using mass action kinetics this translates into the following system of differential equations:

$$
\begin{aligned}
\frac{\mathrm{d}[\mathrm{C}]}{\mathrm{d} t} & =\bar{a}[\mathrm{AC}]-a[\mathrm{~A}][\mathrm{C}]+\bar{b}[\mathrm{BC}]-b[\mathrm{~B}][\mathrm{C}]+2 d\left[\mathrm{C}_{2}\right]-2 \bar{d}[\mathrm{C}]^{2} \\
\frac{\mathrm{d}[\mathrm{AC}]}{\mathrm{d} t} & =a[\mathrm{~A}][\mathrm{C}]-\bar{a}[\mathrm{AC}]+\bar{h}[\mathrm{ABC}]-h[\mathrm{~B}][\mathrm{AC}] \\
\frac{\mathrm{d}[\mathrm{BC}]}{\mathrm{d} t} & =b[\mathrm{~B}][\mathrm{C}]-\bar{b}[\mathrm{BC}]+\bar{g}[\mathrm{ABC}]-g[\mathrm{~A}][\mathrm{BC}] \\
\frac{\mathrm{d}[\mathrm{ABC}]}{\mathrm{d} t} & =g[\mathrm{~A}][\mathrm{BC}]+h[\mathrm{~B}][\mathrm{AC}]-(\bar{g}+\bar{h}+r)[\mathrm{ABC}] \\
\frac{\mathrm{d}\left[\mathrm{C}_{2}\right]}{\mathrm{d} t} & =\bar{d}[\mathrm{C}]^{2}-d\left[\mathrm{C}_{2}\right]+r[\mathrm{ABC}]
\end{aligned}
$$

For the total template concentration

$$
c=[\mathrm{C}]+[\mathrm{AC}]+[\mathrm{BC}]+[\mathrm{ABC}]+2\left[\mathrm{C}_{2}\right] .
$$

we obtain the simple growth law

$$
\frac{\mathrm{d} c}{\mathrm{~d} t}=r[\mathrm{ABC}] .
$$

The quasi-steady state approximation (QSSA), see e.g. [101,11] can now be used to express the concentrations of intermediate products in terms of the free template 
concentration. The total template concentration can also be represented in this way:

$$
\begin{aligned}
& c=[\mathrm{C}]\left[\left(1+\frac{a[\mathrm{~A}]}{\bar{a}+h[\mathrm{~B}]}+\frac{b[\mathrm{~B}]}{\bar{b}+g[\mathrm{~A}]}+Z\right)\left(\frac{\bar{h}}{\bar{a}+h[\mathrm{~B}]}+\frac{\bar{g}}{\bar{b}+g[\mathrm{~A}]}+1+\frac{2 r}{d}\right)\right]+[\mathrm{C}]^{2} \frac{2 \bar{d}}{d} \\
& Z=\frac{[A][B](g b(\bar{a}+h[\mathrm{~B}])+h a(\bar{b}+g[\mathrm{~A}]))}{[\mathrm{A}] g \bar{h}(\bar{b}+g[\mathrm{~A}])+[\mathrm{B}] h \bar{g}(\bar{a}+h[\mathrm{~B}])-(\bar{g}+\bar{h}+r)(\bar{a}+h[\mathrm{~B}])(\bar{b}+g[\mathrm{~A}])}
\end{aligned}
$$

Thus the total template concentration is related to the free template concentration by the quadratic equation

$$
c=X[\mathrm{C}]+Y[\mathrm{C}]^{2} \quad[\mathrm{C}]=\frac{\sqrt{1+4 c Y / X^{2}}-1}{2 Y / X}
$$

where $X$ and $Y$ are the complicated functions of the elementary rate constants defined in equ.(18). With the QSSA equ.(17) becomes

$$
\frac{\mathrm{d} c}{\mathrm{~d} t}=r Z[\mathrm{C}] .
$$

This can be rewritten in the form

$$
\frac{\mathrm{d} c}{\mathrm{~d} t}=\alpha c \psi(\beta c) \quad \text { with } \quad \psi(u)=\frac{2}{u}(\sqrt{1+u}-1)
$$

where $\alpha=r Z / X$ and $\beta=4 Y / X^{2}$. The function $\psi$ has the Taylor series expansion $\psi(u)=1-u / 4+u^{2} / 8+\mathcal{O}\left(u^{3}\right)$ for small arguments $u$ and satisfies $\psi(u)=$ $2 / \sqrt{u}+\mathcal{O}\left(u^{-1}\right)$ for large $u$. The interpretation of the parameters $\alpha$ and $\beta$ is straightforward: The Darwinian fitness $\alpha$ describes the growth rate in dilute solution, while $\beta$ describes the strength of product inhibition.

Equ.(21) is surprisingly general. If we consider three or more fragments that have to be ligated together we eventually find the same functional form of the growth law, of course with even more complicated expressions for $\alpha$ and $\beta$.

A quite different mechanism of replication proceeds via DNA triple helices [66], Fig. 9. Here a DNA duplex C.C is replicated by first forming an adduct C.C'DE with triple helix geometry, where the template strand forms standard WatsonCrick pairs, while the fragments $D$ and $E$ are attached via Hoogsteen pairs. The fragments are ligated and then the resulting $\mathrm{C}^{-C^{\prime} \mathrm{C}}$ complex dissociates along the weaker Hoogsteen pairs. Finally, the single stranded template sequence is ligated with fragments of its complements and forms a copy of the original duplex DNA. The reaction mechanism can be summarized as follows

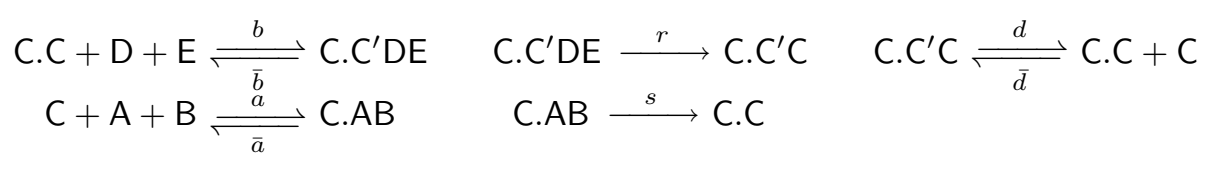

In this simple form the mechanism works only with palindromic DNA since the original and the copied C.C complex are reversed. One can easily envision another round of triple helix formation and dissociation, however. For the purpose of the 


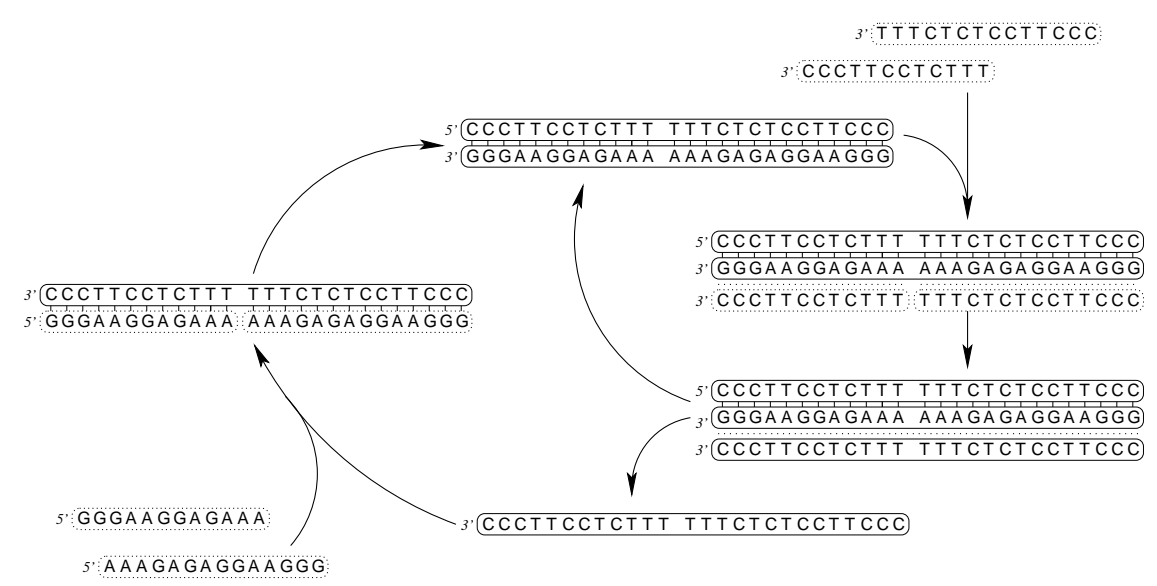

Fig. 9. Self-replication of duplex DNA via a triple-helix stage. In the experiment by Li \& Nicolaou [66] a palindromic DNA template was used. In the general case a suitable second cycle would be necessary. Watson-Crick base pairs are indicated by short lines, Hoogsteen pairs are shown as dots.

kinetic analysis we stick to the palindromic case. The kinetic differential equations read

$$
\begin{aligned}
\frac{\mathrm{d}[\mathrm{C} . \mathrm{C}]}{\mathrm{d} t} & =-b[\mathrm{C} . \mathrm{C}][\mathrm{D}][\mathrm{E}]+\bar{b}\left[\mathrm{C} . \mathrm{C}^{\prime} \mathrm{DE}\right]+s[\mathrm{C} . \mathrm{AB}]+d\left[\mathrm{C} . \mathrm{C}^{\prime} \mathrm{C}\right]-\bar{d}[\mathrm{C} . \mathrm{C}][\mathrm{C}] \\
\frac{\mathrm{d}\left[\mathrm{C} . \mathrm{C}^{\prime} \mathrm{DE}\right]}{\mathrm{d} t} & =b[\mathrm{C} . \mathrm{C}][\mathrm{D}][\mathrm{E}]-\bar{b}\left[\mathrm{C} . \mathrm{C}^{\prime} \mathrm{DE}\right]-r\left[\mathrm{C} . \mathrm{C}^{\prime} \mathrm{DE}\right] \\
\frac{\mathrm{d}\left[\mathrm{C} . \mathrm{C}^{\prime} \mathrm{C}\right]}{\mathrm{d} t} & =r\left[\mathrm{C} . \mathrm{C}^{\prime} \mathrm{DE}\right]-d\left[\mathrm{C} . \mathrm{C}^{\prime} \mathrm{C}\right]+\bar{d}[\mathrm{C}][\mathrm{C} . \mathrm{C}] \\
\frac{\mathrm{d}[\mathrm{C}]}{\mathrm{d} t} & =d\left[\mathrm{C} \cdot \mathrm{C}^{\prime} \mathrm{C}\right]-\bar{d}[\mathrm{C}][\mathrm{C} . \mathrm{C}]-a[\mathrm{C}][\mathrm{A}][\mathrm{B}]+\bar{a}[\mathrm{C} . \mathrm{AB}] \\
\frac{\mathrm{d}[\mathrm{C} \cdot \mathrm{AB}]}{\mathrm{d} t} & =a[\mathrm{C}][\mathrm{A}][\mathrm{B}]-\bar{a}[\mathrm{C} \cdot \mathrm{AB}]-s[\mathrm{C} \cdot \mathrm{AB}]
\end{aligned}
$$

and we have the following expression for the total template concentration

$$
c=[C]+[C \cdot A B]+2[C . C]+2\left[C \cdot C^{\prime} D E\right]+3\left[C \cdot C^{\prime} C\right] .
$$

The net growth law is therefore

$$
\dot{c}=s[\mathrm{C} \cdot \mathrm{AB}]+r\left[\mathrm{C} \cdot \mathrm{C}^{\prime} \mathrm{DE}\right] .
$$

Using the quasi-steady state approximation we obtain

$$
\dot{c}=2 \frac{a s}{\bar{a}+s}[\mathrm{~A}][\mathrm{B}][\mathrm{C}]
$$

and $c=X[\mathrm{C}]+Y[\mathrm{C}]^{2}$ with

$$
X=1+\frac{a}{\bar{a}+s}\left(1+2 \frac{s}{r}+3 \frac{s}{d}\right)[\mathrm{A}][\mathrm{B}]+2 \frac{(\bar{b}+r) a s}{(\bar{a}+s) b r} \frac{[\mathrm{A}][\mathrm{B}]}{[\mathrm{D}][\mathrm{E}]}
$$




$$
Y=3 \frac{\bar{d}}{d} \frac{(\bar{b}+r) a s}{(\bar{a}+s) b r} \frac{[\mathrm{A}][\mathrm{B}]}{[\mathrm{D}][\mathrm{E}]}
$$

The over-all kinetics of equ.(22) is therefore again described by equ.(21), this time with the parameters $\alpha=2 a s[\mathrm{~A}][\mathrm{B}] /[X(\bar{a}+s)]$ and $\beta=4 Y / X^{2}$.

Let us now turn to the competition of different strains of templates $\left[C_{k}\right]$. In [141] we show that in the absence of direct interactions between the templates and with "correct instruction", i.e., in the absence of mutation, the evolution of a mixture of templates is described by the replicator equation

$$
\dot{x}_{k}=x_{k}\left(\alpha_{k} \psi\left(c_{0} \beta_{k} x_{k}\right)-\Phi\right)
$$

This follows from equ.(21) and a transformation to relative concentrations. In addition one obtains an equation for the total concentration $c_{0}$, which we assume here to be regulated such that it varies only slowly. In this case one can regard $c_{0}$ as an additional tunable parameter.

The analysis of equ.(27) is in essence based on Theorem A from [42], which can be restated in the following form

Theorem 5.1. (i) There is a unique fixed point $\hat{x}$ which is the $\omega$-limit of all orbits in the interior of the simplex $S_{n}$. (ii) If $\hat{x}$ lies in the interior of a face, then it is also the $\omega$-limit of all orbits in the interior of its face. (iii) If the species are labeled such that $\alpha_{1} \geq \alpha_{2} \geq \ldots \alpha_{n}$, then there is an index $m \geq 1$ such that $\hat{x}$ is of the form $\hat{x}_{i}>0$ if $i \leq m$ and $\hat{x}_{i}=0$ for $i>m$. (iv) If $\min \left\{\beta_{k}\right\}$ is large enough, then $m=n$ and $\hat{x}$ is a uniquely determined interior fixed point.

It is shown in [47] that equ.(27) is a Shashahani gradient system. Furthermore, $V(x)=\prod_{k} x_{k}^{z_{k}}$ is a Ljapunov function, when $z_{k}$ are the coordinates of the globally stable fixed point.

It is not hard to verify that the condition for survival of species $k$, item (iii) in Theorem 5.1, is explicitly

$$
\alpha_{k}>\Phi(\hat{x}) .
$$

If we sort the replicating species according to decreasing values of the Darwinian fitness parameters, $\alpha_{1} \geq \alpha_{2} \geq \ldots \geq \alpha_{n}$, then there is an index $m$ such that $\hat{x}$ is of the form $\hat{x}_{k}>0$ if $k \leq m$ and $\hat{x}_{k}=0$ for $k>m$. In other words, $m$ species survive while the $n-m$ least efficient replicators die out. This behavior is completely analogous to the reversible exponential competition model discussed in [97], where the rate constants $a_{k}$ play the role of our Darwinian fitness parameters $\alpha_{k}$.

The threshold value $\Phi(\hat{x})$ can be computed explicitly in the form

$$
\Phi(\hat{x})=\frac{1}{c_{0}} \sum_{k}^{m} \frac{\alpha_{k}}{\beta_{k}}\left[\sqrt{1+c_{0}\left(\sum_{k}^{m} \frac{\alpha_{k}^{2}}{\beta_{k}}\right) /\left(\sum_{k}^{m} \frac{\alpha_{k}}{\beta_{k}}\right)^{2}}-1\right]
$$

where the sum runs over the surviving species only. It is interesting to note that the Darwinian fitness parameters $\alpha_{k}$ determine the order in which species reach 


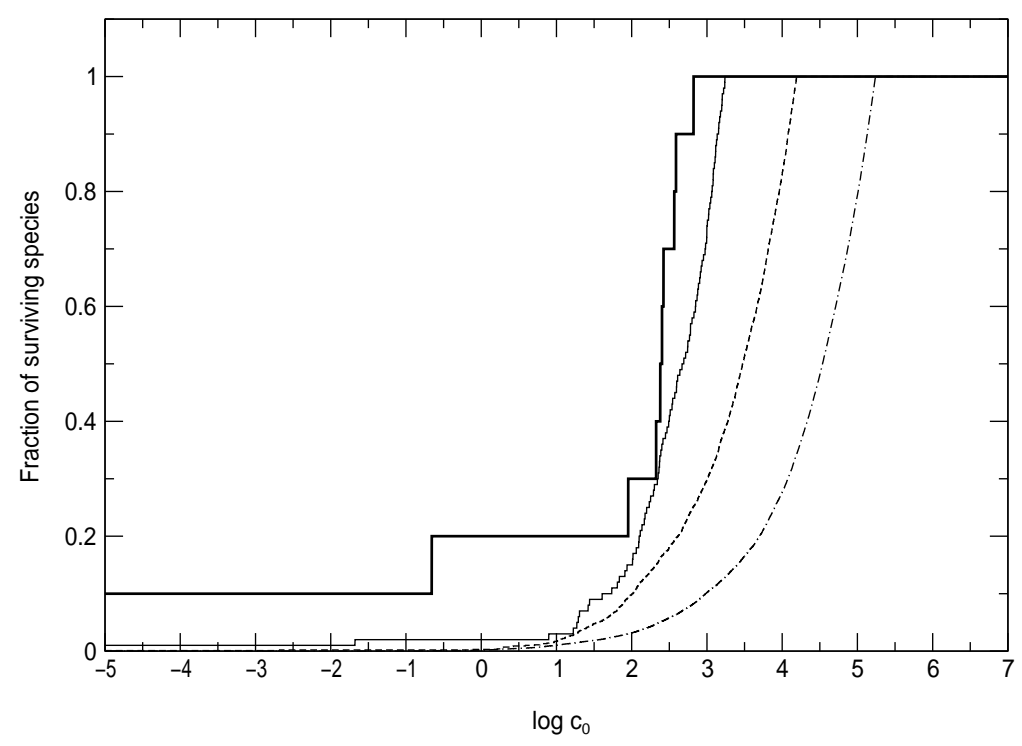

Fig. 10. Fraction of surviving species as a function of the total concentration $c$ for $n=10,100$, 1000, 10000 species (from left to right) with $\alpha_{k} \sim \exp (-k / n)$ and $\beta_{k}$ chosen from Uniform[0,1]. Data are taken from [113].

extinction, whereas the concentration-dependent values $\beta_{k} c_{0}$ collectively influence the flux term and hence set the "extinction threshold". In contrast to Szathmáry's model equation [123] the extended replicator kinetics leads to both competitive selection and coexistence of replicators depending on total concentration and kinetic constants. For small $c_{0}$ it behaves like a quasispecies model, while product inhibition and therefore unconditional coexistence dominates in a "thick soup", Fig. 10.

Systems of competing species replicating via template-directed ligation therefore can meet two of the criteria required for effective evolution: (i) strong selection leading to the extinction of some or even almost all species, and (ii) susceptibility to invasion by new, advantageous species. The origin of life requires a mechanism of chemical replication in which strong selection enables some species to outgrow others, the "losers" which die out. On the other hand, the coexistence of more than one "master sequence" is required for functional specialization and cooperation to emerge because of the limitations on heritable information that are imposed by the error threshold. Replicators which reproduce through duplex formation utilizing a Michaelis-Menten type of mechanism fulfil these conditions much more easily than replicators needing the strong and specific catalytic interactions characteristic of hypercyclic cooperation $[28,119]$. Therefore they are good candidates for the first molecules which may have been selectively amplified in the prebiotic environment. 


\section{Higher Order Systems}

In this section we briefly consider plausible molecular mechanisms for ligation based replication involving both a template and a second "catalyst" that acts as a "replicase ribozyme". Experimentally such a system is at least reasonable since multiple helices are well known structures in both nucleic acids and coiled-coil peptides. Alternatively, one may think of the catalyst as a version of Johnston's [57] RNA replicase shown in Fig. 1a.

The generic features of the resulting reaction networks are largely unexplored despite some efforts towards understanding the dynamics of chemically realistic models of "prebiotic" replication in [41]. On the other hand, the limiting case of strong selection is well understood. When complex formation and product inhibition play no role the dynamics is described by the second order replicator equations which we have considered in section 3. In the spirit of the previous section we consider a template species $C$ that can be obtained by the concatenation (ligation) of two building blocks $A$ and $B$ provided in the environment. Again, a generalization to multiple building blocks is straightforward even though explicit expressions for the aggregate parameters in terms of the elementary rate constants become too complicated to be useful. We restrict ourselves to a rather qualitative discussion here, more technical details can be found in [112].

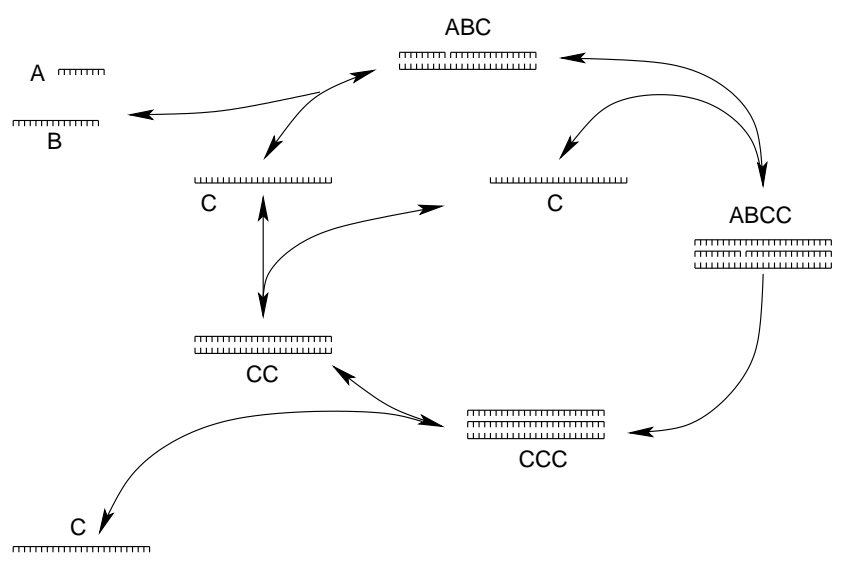

Fig. 11. Scheme of a ligation-replication mechanism with higher order catalysis.

The crucial step of the replication mechanism is the formation of an intermediate species $A B C C$ which we may visualize as a triple helix consisting of the template $C$, the building material $A$ and $B$ properly aligned along $C$ so that the ligation of the parts $A$ and $B$ can readily proceed, and an additional copy of $C$ that might be thought either as a specific catalyst that is involved in the ligation reaction, or its role might be seen in facilitating the release of the product $C$. In either case, we consider the reaction scheme of Fig. 11. 
It is instructive to compare the reaction scheme in Fig. 11 with the triple-helix ligation model of Li \& Nicolaou in Fig. 9. The main difference is that in the present model the catalyst $[C]$ is attached after the ligation complex $[A B C]$ is formed. The consequences of this difference on the dynamical behavior are rather dramatic. A tedious computation [112] yields

$$
\begin{gathered}
c=\frac{p_{4}[\mathrm{C}]^{4}+p_{3}[\mathrm{C}]^{3}+p_{2}[\mathrm{C}]^{2}+p_{1}[\mathrm{C}]}{q_{2}[\mathrm{C}]^{2}+q_{1}[\mathrm{C}]+q_{0}} \\
\frac{\mathrm{d} c}{\mathrm{~d} t}=\frac{\mathrm{d}[\mathrm{C}]}{\mathrm{d} t}=a[\mathrm{ABCC}]=[\mathrm{A}][\mathrm{B}][\mathrm{C}]^{2} \frac{\alpha+\gamma[\mathrm{C}]}{\beta+\delta[\mathrm{C}]}
\end{gathered}
$$

where the QSSA is used to obtain the last equality. All coefficients here are complicated combinations of the elementary rate constants, all of which are positive. For small values of $c \ll 1$ we have $c \approx p_{1}[\mathrm{C}] / q_{0}$ and hence

$$
\dot{c} \sim \frac{q_{0}^{2} \alpha}{p_{1}^{2} \beta}[\mathrm{A}][\mathrm{B}] c^{2},
$$

i.e., we have second order autocatalytic or hyperbolic growth.

For large values of $c$ we have $c \approx\left(p_{4} / q_{1}\right)[C]^{3}$ and hence

$$
\dot{c} \sim \sqrt[3]{\frac{q_{1}^{2}}{p_{4}^{2}}} \frac{\gamma}{\delta}[\mathrm{A}][\mathrm{B}] c^{2 / 3} .
$$

The concentration grows polynomially in this case.

If $q_{2}[\mathrm{C}]^{2}$ cannot be neglected, we have $c \approx\left(p_{4} / q_{2}\right)[\mathrm{C}]^{2}$ for small values of $c$, and hence

$$
\dot{c} \propto \frac{q_{2} \gamma}{p_{4} \delta} c,
$$

i.e., we observe exponential growth.

Let us now consider the case of multiple templates and assume that each of them catalyzes only its own replication. It can be shown [112] that the relative concentrations again follow a replicator equation, which now is of the form shown in l.h.s. of Fig. 12:

$$
\dot{x}_{k}=x_{k}\left[\varphi_{k}\left(x_{k} ; c_{0}\right)-\Phi\right]
$$

with the growth functions $\varphi_{k}$ of the form

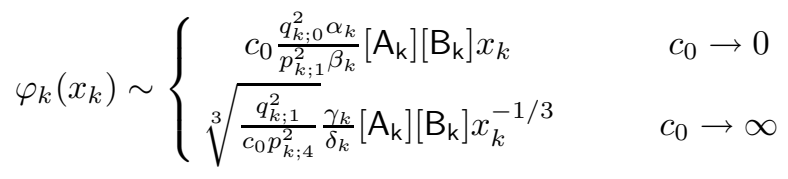

As a consequence, we recover two well studied models as limiting cases: In dilute solution, $c_{0} \rightarrow 0$, we obtain Schlögl's model of competing exponentially growing autocatalysts [90], in saturated solution, $c_{0} \rightarrow \infty$, we recover Szathmáry's model of parabolic growth, equ. (14) [123], with the exponent $a=2 / 3$. The transition between the two limiting cases proceeds via the series of saddle-node bifurcations 

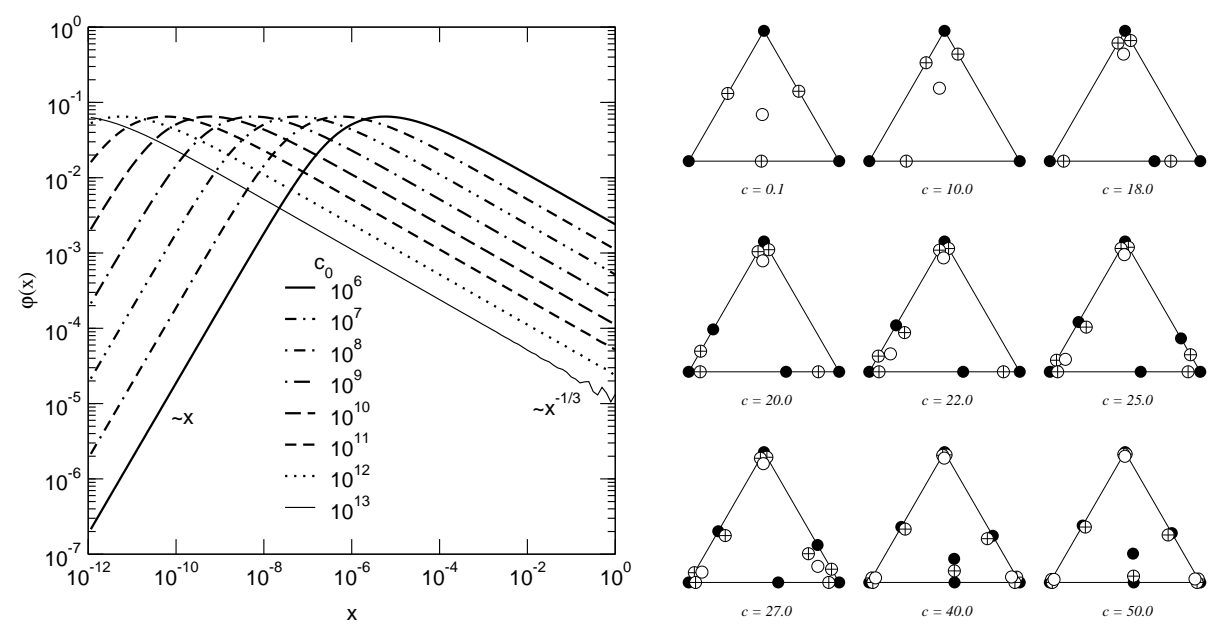

Fig. 12. L.h.s .: Growth function $\varphi$, R.h.s.: Bifurcation diagram as a function of $c_{0}$.

shown on the r.h.s. of Fig. 12. Similarly, one obtains second order replicator equations in the case of cross-catalysis in dilute solution. More details can be found in [112].

\section{Discussion}

Template induced replication is the basis of multiplication and reproduction in nature and in vitro, hence a detailed understanding of its mechanisms in terms of chemical reaction kinetics is an important facet of understanding evolution at the molecular level. Simple single step mechanisms of independent replication lead to exponential growth while the explicit consideration of dissociation of the replication complex reduces the net rate of synthesis and yields parabolic growth. Only a detailed mechanistic analysis reveals that both types of behavior result from the same chemically plausible mechanisms, albeit under different conditions.

Evolution is limited in both cases. An error threshold limits the amount of heritable information in the selection dominated exponential growth regime, while parabolic growth essentially switches off selection because all replicators coexist. Cooperatively coupled replicators, such as the hypercycle [28], are one possibility to overcome the error threshold. These networks, however, are susceptible to parasitic mutants and invaders. Furthermore, it is not clear at this point that uncatalyzed replication can sustain RNAs that are large enough to efficiently assist in RNA replication.

Models with significant product inhibition bridge this gap. A "survival threshold" allows the coexistence of not only the fittest molecule but of an ensemble of possibly unrelated fit replicators. These might collectively accumulate sufficient information for active catalysts and hence lead to the onset of a positive feedback loop that leads to the evolution of efficient ribozymes. In this scenario selection is 
still an important force that removes unfit variants from the pool. On the other hand, it appears to at least alleviate the parasite problem that plagues the second order replicator models.

Of course, a homogeneous "soup" environment is not the only possibility. The idea to consider a spatial organization as a means of overcoming the parasite problem was introduced by Boerlijst \& Hogeweg [9], who investigated hypercycles with parasites using a two-dimensional cellular automaton. The authors observed spirals that stabilized the hypercycles in competition with the parasite. A similar model based on partial differential equations produced comparable results [120]. Later work showed, however, that the stabilizing effect caused by the spiral patterns is highly model-dependent [18]. Coexistence of quasispecies as a consequence of spatial structure and limited diffusion is described in [127].

A different scenario is replication in open chaotic flows such as those near hydrothermal vents. The associated fractal dynamics leads to singularly enhanced concentrations, resulting in a growth law for the replicating material of the form

$$
\frac{\mathrm{d}[\mathrm{C}]}{\mathrm{d} t}=-\kappa[\mathrm{C}]+\nu[\mathrm{C}]^{-\beta}
$$

that is completely different from the behavior in homogeneous solution or on a surface $[59,130]$. Similar to models with parabolic growth it leads to coexistence.

Another interesting class of models, which is however beyond the scope of this contribution, assumes that the genetic material is already encapsulated in a protocell. This protocell duplicates with a rate that depends on the type and concentration of the enclosed material. Both the stochastic corrector model [122] and hypercycles in compartments can explain the accumulation of information and show significant resistance against parasites of various kinds, as shown in recent computer simulations [146]. It is interesting to note that the genes that are enclosed in the compartment of the stochastic corrector model form a catalytic network that would be unstable in homogeneous solution. Information accumulation through compartmentalization would therefore seem to require that a very short "genome" can already significantly influence the duplication of the compartment. This possibility is supported to a certain extent by the fact that RNA molecules can specifically interact with phospholipid bilayers and regulate its permeability [134]. The particular RNA molecules described in [134], however, are by far too large to be replicated without enzymes or ribozymes. Once there is a replicase-ribozyme that can replicate itself (which is pre-supposed in both the hypercycle and the stochastic corrector model) there is a selection pressure even in a homogeneous medium to improve the replication accuracy which in turn allows for larger genomes and hence opens up additional possibilities for increasing the accuracy [87]. Compartmentalization per $s e$ is therefore neither necessary nor sufficient to overcome the information bottleneck imposed by the error-threshold. Since it is by no means clear that compartments could facilitate the evolution of the first replicase-ribozyme, we cannot use replication kinetics to deduce whether compartmentalization should have occurred 
before or after the invention of catalyzed replication. The kinetics of uncatalyzed ligation-based replication linked to a compartment will be explored elsewhere [85].

In this contribution we have restricted ourselves to chemical kinetics and its consequences, i.e., to models of "chemical population dynamics". A more complete model of evolution would have to include a description of the mutants that are accessible from a given population [30] and a way to construct the kinetic constants from a mechanistic model. In the simplest cases this leads to the notion of a fitness landscape $[143,144]$ on which the evolving population moves. The properties of this landscape then determine the long-term outcome of evolution, see e.g. [95,94]. While the theory of fitness landscapes [86] is quite well developed, the generalization to networks of interacting replicators [31] has not yet lead to a concise theory of the long term evolution of strongly interacting replicators.

\section{Acknowledgments}

This work was supported in part by the Austrian Fonds zur Förderung der Wissenschaftlichen Forschung, Project No. P-13887-MOB.

\section{References}

[1] Alves D. and Fontanari J.F. Population genetics approach to the quasispecies model. Phys. Rev. E, 54, 4048-4053 (1996).

[2] Alves D. and Fontanari J.F. Error threshold in finite populations. Phys. Rev. E, 57, 7008-7013 (1998).

[3] Amann E. Permanence in Catalytic Networks. Ph.D. thesis, Universität Wien, Institut für Mathematik (1989).

[4] Andrade M.A., Nuño J.C., Morán F., Montero F., and Mptisos G. Complex dynamics of a catalytic network having faulty replication into error-species. Physica $D, \mathbf{6 3}$, 21-40 (1993).

[5] Arneodo A., Coullet P., and Tresser C. Occurence of strange attractors in threedimensional Volterra equations. Phys. Lett. A, 79, 259-263 (1980).

[6] Bachmann P.A., Luisi P.L., and Lang J. Autocatalytic self-replicating micelles as models for prebiotic structures. Nature, 357, 57-59 (1992).

[7] Baskerville S. and Bartel D.P. A ribozyme that ligates RNA to protein. Proc. Natl. Acad. Sci. USA, 99, 9154-9159 (2002).

[8] Biebricher C.K. and Eigen M. Kinetics of RNA replication by $\mathrm{Q} \beta$ replicase. In $R N A$ Genetics. Vol.I: RNA Directed Virus Replication, E. Domingo, J.J. Holland, and P. Ahlquist, eds., pp. 1-21 (CRC Press, Boca Raton, FL, 1988).

[9] Boerlijst M.C. and Hogeweg P. Spiral wave structure in pre-biotic evolution: Hypercycles stable against parasites. Physica D, 48, 17-28 (1991).

[10] Bomze I. Lotka-Volterra equations and replicator dynamics: A two-dimensional classification. Biol. Cybern., 48, 201-211 (1983).

[11] Borghans J.A.M., de Boer R.J., and Segel L.A. Extending the quasi-steady state approximation by changing variables. Bull. Math. Biol., 58, 43-63 (1996).

[12] Brack A., ed. The Molecular Origins of Life: Assembling Pieces of the Puzzle (Cambridge University Press, Cambridge, UK, 1999).

[13] Brannath W. Heteroclinic networks on the tetrahedron. Nonlinearity, 7, 1367-1384 (1994). 
[14] Bresch C., Niesert U., and Harnasch D. Hypercycles, parasites, and packages. J. Theor. Biol., 85, 399-405 (1980).

[15] Campos P.R.A. and Fontanari J.F. Finite-size scaling of the error threshold transition in finite population. J. Phys. A: Math. Gen., 32, L1-L7 (1999).

[16] Campos P.R.A., Fontanari J.F., and Stadler P.F. Error propagation in the hypercycle. Phys. Rev. E, 61, 2996-3002 (2000).

[17] Chaput J.C. and Switzer C. Nonenzymatic oligomerization on templates containing phosphoester-linked acyclic glycerol nucleic acid analogues. J. Mol. Evol., 51, 464470 (2000).

[18] Cronhjort M.B. and Blomberg C. Hypercycles versus parasites in a two dimensional partial differential equations model. J. Theor. Biol., 169, 31-49 (1994).

[19] Dawkins R. The Selfish Gene (Oxford University Press, Oxford, 1976).

[20] Domingo E. Biological significance of viral quasispecies. Viral Hepatitis Rev., 2, 247-261 (1996).

[21] Domingo E. and Holland J.J. RNA virus mutations and fitness for survival. Ann. Rev. Microbiol., 51, 151-178 (1997).

[22] Doudna J.A. and Cech T.R. The chemical repertoire of natural ribozymes. Nature, 418, 222-228 (2002).

[23] Drake J.W. Rates of spontaneous mutation among RNA viruses. Proc. Natl. Acad. Sci. USA, 90, 4171-4175 (1993).

[24] Drake J.W., Charlesworth B., Charlesworth D., and Crow J.F. Rates of spontaneous mutations. Genetics, 148, 1667-1686 (1998).

[25] Eigen M. Selforganization of matter and the evolution of biological macromolecules. Naturwissenschaften, 58, 465-523 (1971).

[26] Eigen M., McCaskill J., and Schuster P. Molecular Quasi-Species. J. Phys. Chem., 92, 6881-6891 (1988).

[27] Eigen M., McCaskill J.S., and Schuster P. The molecular quasi-species. Adv. Chem. Phys., 75, 149-263 (1989).

[28] Eigen M. and Schuster P. The Hypercycle (Springer-Verlag, New York, Berlin, 1979).

[29] Famulok M., Nowick J., and Rebek Jr. J. Self-Replicating Systems. Act. Chim. Scand., 46, 315-324 (1992).

[30] Fontana W. and Schuster P. Continuity in evolution: On the nature of transitions. Science, 280, 1451-1455 (1998).

[31] Forst C.V. Molecular evolution of catalysis. J. Theor. Biol., 205, 409-431 (2000).

[32] Fox S.W. and Dose H. Molecular Evolution and the Origin of Life (Academic Press, New York, 1977).

[33] Freedman H. and Moson P. Persistence definitions and their connections. Proc. Amer. Math. Soc., 109, 1025-1033 (1990).

[34] Freedman H. and Waltman P. Mathematical analysis of some three-species foodchain models. Math. Biosc., 33, 257-276 (1977).

[35] Freeland S.J., Knight R.D., and Landweber L.F. Do proteins predate DNA? Science, 286, 690-692 (1999).

[36] Gesteland R.F. and Atkins J.F., eds. The RNA World (Cold Spring Harbor Laboratory Press, Plainview, NY, 1993).

[37] Gilbert W. The RNA world. Nature, 319, 618 (1986).

[38] Gilpin M.E. Spiral chaos in a predator prey system. Amer. Nat., 133, 306-308 (1978).

[39] Happel R. and Stadler P.F. The evolution of diversity in replicator networks. J. Theor. Biol., 195, 329-338 (1998).

[40] Happel R. and Stadler P.F. Autocatalytic replication in a CSTR and constant or- 
ganization. J. Math. Biol., 38, 422-434 (1999).

[41] Hecht R., Happel R., Schuster P., and Stadler P.F. Autocatalytic networks with intermediates I: Irreversible reactions. Math. Biosc., 140, 33-74 (1997).

[42] Hofbauer J. On the occurrence of limit cycles in Volterra-Lotka equations. Nonlin. Anal., 5, 1003-1007 (1981).

[43] Hofbauer J. The selection mutation equation. J. Math. Biol., 23, 41-53 (1985).

[44] Hofbauer J. Saturated equilibria, permanence, and stability for ecological systems. In Mathematical Ecology: Proceedings on the Second Autumn Course on Mathematical Ecology, Trieste, Italy, T.G. Hallham, L.J. Gross, and S.A. Lewin, eds., pp. 625-642 (World Scientific, Singapore, 1986).

[45] Hofbauer J. Competitive exclusion of disjoint hypercycles. Z. Phys. Chem., 216, 35-39 (2002).

[46] Hofbauer J. and Sigmund K. Permanence for replicator equations. In Lecture Notes Econ. Math., volume 287, pp. 131-146 (Springer-Verlag, Berlin, Heidelberg, New York, 1987).

[47] Hofbauer J. and Sigmund K. Dynamical Systems and the Theory of Evolution (Cambridge University Press, Cambridge U.K., 1988).

[48] Hofbauer J. and Sigmund K. Evolutionary Games and Population Dynamics (Cambridge University Press, Cambridge U.K., 1998).

[49] Hofbauer J. and So J. Multiple limit cycles for three-dimensional Lotka-Volterra equations. Appl. Math. Lett., 7, 65-70 (1994).

[50] Hutson V. and Moran W. Persistence of species obeying difference equations. Bull. Math. Biol., 15, 203-212 (1982).

[51] Hutson V. and Schmitt K. Permanence and the dynamics of biological systems. Math. Biosc., 111, 1-71 (1992).

[52] Illangasekare M. and Yarus M. A tiny RNA that catalyzes both aminoacyl-RNA and peptidyl-RNA synthesis. RNA, 5, 1482-1489 (1999).

[53] Jadhav V.R. and Yarus M. Coenzymes as coribozymes. Biochimie, ???, ??? (2002). In press, doi 10.1016/S0300-9084(02)01404-9.

[54] James K.D. and Ellington A.D. The fidelity of template-directed oligonucleotide ligation and the inevitability of polymerase function. Orig. Life Evol. Biosph., 29, 375-390 (1999).

[55] Jansen W. A permanence theorem for replicator and Lotka-Volterra systems. J. Math. Biol., 25, 411-422 (1986).

[56] Jeffares Daniel C. andPoole A.M. and Penny D. Relics from the RNA world. J. Mol. Evol., 46, 18-36 (1998).

[57] Johnston W.K., Unrau P.J., Lawrence M.J., Glasner M.E., and Bartel D.P. RNAcatalyzed RNA polymerization: Accurate and general RNA-templated primer extension. Science, 292, 1319-1325 (2001).

[58] Joyce G.F. The antiquity of RNA-based evolution. Nature, 418, 214-221 (2002).

[59] Károlyi G., Péntek Á., Scheuring I., Tél T., and Toroczkai Z. Chaotic flow: the physics of species coexistence. Proc. Natl. Acad. Sci., 97, 13661-13665 (2000).

[60] Keefe A.D. and Miller S.L. Are polyphosphates or phosphate esters prebiotic reagents? J. Mol. Evol., 41, 693-702 (1995).

[61] Kumar R.K. and Yarus M. RNA-catalyzed amino acid activation. Biochemistry, 40, 6998-7004 (2001).

[62] Lee D.H., Granja J.R., Martinez J.A., Severin K., and Ghadiri M.R. A selfreplicating peptide. Nature, 382, 525-528 (1996).

[63] Lee D.H., Severin K., Yokobayashi Y., and Ghadiri M.R. Emergence of symbiosis in peptide self-replication through a hypercyclic network. Nature, 390, 591-594 (1997). 
[64] Lee N., Bessho Y., Wei K., Szostak J.W., and Suga H. Ribozyme-catalyzed tRNA aminoacylation. Nat. Struct. Biol, 7, 28-33 (2000).

[65] Leung D.W., Chen E., and Goeddel D.V. A method for random mutagenesis of a defined DNA segment using a modified polymerase chain reaction. Technique, $\mathbf{1}$, 11-15 (1989).

[66] Li T. and Nicolaou K.C. Chemical self-replication of palindromic duplex DNA. Nature, 369, 218-221 (1994).

[67] Martinez M.A., Vartanian J.P., and Wain-Hobson S. Hypermutagenesis of RNA using human immunodeficiency virus type 1 reverse transcriptase and biased dNTP concentrations. Proc. Natl. Acad. Sci. USA, 91, 11787-11791 (1994).

[68] Mason S.F. Chemical evolution. Origin of the elements, molecules, and living systems (Clarendon Press, Oxford (UK), 1991).

[69] Mavelli F. and Luisi P.L. Autopoietic self-reproducing vesicles: A simplified kinetic model. J. Phys. Chem., 100, 16600-16607 (1996).

[70] May R.M. and Nowak M.A. Superinfection, metapopulation dynamics, and the evolution of diversity. J. Theor. Biol., 170, 95-114 (1994).

[71] May R.M. and Nowak M.A. Coinfection and the evolution of parasite virulence. Proc. R. Soc. London B, 261, 209-215 (1995).

[72] Maynard Smith J. Hypercycles and the origin of life. Nature, 280, 445-446 (1979).

[73] McCaskill J.S. A localisation threshhold for macromolecular quasispecies from continuously distributed replication rates. J. Chem. Phys., 80, 5194 (1984).

[74] McCaskill J.S. Spatially resolved in vitro molecular ecology. Biophys Chem, 66, 145-158 (1997).

[75] Mills D.R., Peterson R.L., and Spiegelman S. An extracellular Darwinian experiment with a self-duplicating nucleic acid molecule. Proc. Natl. Acad. Sci. USA, 58, 217224 (1967).

[76] Moore P.B. and Steitz T.A. The involvement of RNA in ribosome function. Nature, 418, 229-235 (2002).

[77] Nelson K.E., Levy M., and Miller S.L. Peptide nucleic acids rather than RNA may have been the first genetic molecule. Proc. Natl. Acad. Sci. USA., 97, 3868-3871 (2000).

[78] Nisbet E.G. and Sleep N.H. The habitat and nature of early life. Nature, 409, 1083-1091 (2001).

[79] Novick J.S., Feng Q., and Tjivikua T. Kinetic studies and modeling of a selfreplicating system. J. Amer. Chem. Soc., 113, 8831-8838 (1991).

[80] Nowak M. and Schuster P. Error thresholds of replication in finite populations. Mutation frequencies and the onset of Muller's ratchet. J. Theor. Biol., 137, 375395 (1989).

[81] Nuño J.C., Andrade M.A., Morán F., and Montero F. A model of an autocatalytic network formed by error-prone self-replicative species. Bull. Math. Biol., 55, 385415 (1993).

[82] Orgel L.E. The origin of life - a review of facts and speculations. Trends Biochem. Sci., 23, 491-495 (1999).

[83] Pitsch S., Krishnamurthy R., Bolli M., Wendeborn S., Holzner A., Minton M., Lesueur C., Schlonvogt I., Jaun B., and Eschenmoser A. Pyranosyl-RNA (p-RNA): base-pairing selectivity and potential to replicate. Helv. Chim. Acta, 78, 1621-1635 (1995).

[84] Poole A., Penny D., and Sjäberg B.M. Methyl-RNA: an evolutionary bridge between RNA and DNA? Chem. \& Biol., 7, R207-R216 (2000).

[85] Rasmussen S., Chen L., Stadler B.M.R., and Stadler P.F. Proto-organism kinetics: 
Evolutionary dynamics of lipid aggregates with genes and metabolism. Orig. Life Evol. Biosph. (2002). Submitted.

[86] Reidys C.M. and Stadler P.F. Combinatorial landscapes. SIAM Review, 44, 3-54 (2002).

[87] Scheuring I. Avoiding catch-22 of early evolution by stepwise increase in copying fidelity. Selection, 1, 135-145 (2000).

[88] Scheuring I. and Szathmáry E. Survival of replicators with parabolic growth tendency and exponential decay. J. Theor. Biol., 212, 99-105 (2001).

[89] Schidlowski M. A 3,800 million-year old record of life from carbon in sedimentary rocks. Nature, 333, 313-318 (1988).

[90] Schlögl F. Chemical reaction models for non-equilibrium phase transitions. Z Physik, 253, 147-161 (1972).

[91] Schnabl W., Stadler P.F., Forst C., and Schuster P. Full characterization of a strange attractor. Physica D, 48, 65-90 (1991).

[92] Schöning K.U., Scholz P., Guntha S., Wu X., Krishnamurthy R., and Eschenmoser A. Chemical etiology of nucleic acid structure: the $\alpha$-threofuranosyl-(3' $\left.\rightarrow 2^{\prime}\right)$ oligonucleotide system. Science, 290, 1347-1351 (2000).

[93] Schopf J.W. and Packer B.M. Early Archean (3.3 billion to 3.5 billion year old) microfossils from Warrawoona Group, Australia. Science, 237, 70-73 (1987).

[94] Schuster P. Landscapes and molecular evolution. Physica D, 107, 351-365 (1997).

[95] Schuster P., Fontana W., Stadler P.F., and Hofacker I.L. From sequences to shapes and back: A case study in RNA secondary structures. Proc. Roy. Soc. Lond. B, 255, 279-284 (1994).

[96] Schuster P. and Sigmund K. Replicator dynamics. J. Theor. Biol., 100, 533-538 (1983).

[97] Schuster P. and Sigmund K. Dynamics of evolutionary optimization. Ber. BunsenGesellsch. Phys. Chem., 89, 668-682 (1985).

[98] Schuster P., Sigmund K., and Wolff R. Dynamical systems under constant organization III: Cooperative and competitive behaviour of hypercycles. J. Diff. Eqns., 32, 357-368 (1979).

[99] Schuster P., Sigmund K., and Wolff R. Mass action kinetics of selfreplication in flow reactors. J. Math. Anal. Appl., 78, 88-112 (1980).

[100] Schwartz A.W. Speculation on the RNA precursor problem. J. Theor. Biol., 187, 523-527 (1997).

[101] Segel L.A. and Slemrod M. The quasi-steady state assumption: a case study in perturbation. SIAM Rev., 31, 446-477 (1989).

[102] Segré D., Ben-Eli D., Deamer D.W., and Lancet D. The lipid world. Orig. Life. Evol. Biosph., 31, 119-145 (2001).

[103] Segré D., Ben-Eli D., and Lancet D. Compositional genomes: Prebiotic information transfer in mutually catalytic noncovalent assemblies. Proc. Natl. Acad. Sci. USA, 97 (2000).

[104] Segré D., Shenav B., Kafri R., and Lancet D. The molecular roots of compositional inheritance. J. Theor. Biol., 213, 481-491 (2001).

[105] Shapiro R. Prebiotic cytosine synthesis: a critical analysis and implications for the origin of life. Proc. Natl. Acad. Sci. USA, 96, 4396-4401 (1999).

[106] Sievers D. and von Kiedrowski G. Self-replication of complementary nucleotidebased oligomers. Nature, 369, 221-224 (1994).

[107] Sigmund K. and Schuster P. Permanence and uninvadability for deterministic population models. In Stochastic Phenomena and Chaotic Behaviour in Complex Systems, P. Schuster, ed., volume 21 of Synergetics, pp. 186-205 (Springer-Verlag, New 
York, 1984).

[108] Sleep N.H., Zahnle K.J., Kasting J.F., and Morowitz H.J. Annihilation of ecosystems by large asteroid impacts on the early Earth. Nature, 342, 139-142 (1989).

[109] Spiegelman S. An approach to the experimental analysis of precellular evolution. Quart. Rev. Biophys., 4, 213-253 (1971).

[110] Stadler B.M.R. Segregation distortion and heteroclinic cycles. J. Theor. Biol., 183, 363-379 (1996).

[111] Stadler B.M.R. and Stadler P.F. Dynamics of small autocatalytic reaction networks III: Monotonous growth functions. Bull. Math. Biol., 53, 469-485 (1991).

[112] Stadler B.M.R., Stadler P.F., and Schuster P. Dynamics of autocatalytic replicator networks based on higher order ligation reactions. Bull. Math. Biol., 62, 1061-1086 (2000).

[113] Stadler B.M.R., Stadler P.F., and Wills P.R. Evolution in systems of ligation-based replicators. Z. Phys. Chem., 21-33, 216 (2001).

[114] Stadler P.F. and Happel R. The probability for permanence. Math. Biosc., 113, 25-50 (1993).

[115] Stadler P.F. and Nuño J.C. The influence of mutation on autocatalytic reaction networks. Math. Biosci., 122, 127-160 (1994).

[116] Stadler P.F., Schnabl W., Forst C.V., and Schuster P. Dynamics of small autocatalytic reaction networks II: Replication, mutation, and catalysis. Bull. Math. Biol., 57, 21-61 (1995).

[117] Stadler P.F. and Schuster P. Dynamics of small autocatalytic reaction networks I: Bifurcations, permanence and exclusion. Bull. Math. Biol., 52, 485-508 (1990).

[118] Stadler P.F. and Schuster P. Mutation in autocatalytic networks - an analysis based on perturbation theory. J. Math. Biol., 30, 597-631 (1992).

[119] Stadler P.F. and Schuster P. Permanence of sparse autocatalytic networks. Math. Biosc., 131, 111-134 (1996).

[120] Streissler C. Autocatalytic networks under diffusion. Ph.D. thesis, Universität Wien, Austria (1992). Ph.D. Thesis.

[121] Swetina J. and Schuster P. Self-replication with errors - A model for polynucleotide replication. Biophys. Chem., 16, 329-345 (1982).

[122] Szathmáry E. and Demeter L. Group selection of early replicators and the orgin of life. J. Theor. Biol., 128, 463-486 (1987).

[123] Szathmáry E. and Gladkih I. Sub-exponential growth and coexistence of nonenzymatically replicating templates. J. Theor. Biol., 138, 55-58 (1989).

[124] Szathmáry E. and Maynard-Smith J. From replicators to reproducers: the first major transitions leading to life. J. Theor. Biol., 187, 555-571 (1997).

[125] Tarazona P. Error-tresholds for molecular quasi-species as phase transitions: From simple landscapes to spinglass models. Phys. Rev. A[15], 45, 6038-6050 (1992).

[126] Taylor P.D. and Jonker L.B. Evolutionary stable strategies and game dynamics. Math.Biosc., 40, 145-156 (1978).

[127] Tereshko V. Selection and coexistence by reaction-diffusion dynamics in fitness landscapes. Phys. Let. A, 260, 522-527 (1999).

[128] Tilman D. Competition and biodiversity in spatially structured habitats. Ecology, 75, 2-16 (1994).

[129] Tjivikua T., Ballester P., and Rebek Jr. J. A self-replicating system. J. Am. Chem. Soc., 112, 1249-1250 (1990).

[130] Toroczkai Z., Károlyi G., Péntek Á., Tél T., and Grebogi C. Advection of active particles in open chaotic flows. Phys. Rev. Lett., 80, 500-503 (1998).

[131] Unrau P.J. and Bartel D.P. RNA-catalysed nucleotide synthesis. Nature, 395, 260- 
263 (1998).

[132] Vance R.R. Predation and resource partitioning in one predator - two prey model communities. Amer. Nat., 112, 797-813 (1978).

[133] Veronese A. and Luisi P.L. An autocatalytic reaction leading to spontaneously assembled phosphatidyl nucleoside giant vesicles. J. Amer. Chem. Soc., 120, 26622663 (1998).

[134] Vlassov A., Khvorova A., and Yarus M. Binding and disruption of phospholipid bilayers by supramolecular RNA complexes. Proc. Natl. Acad. Sci. USA, 98, 77067711 (2001).

[135] von Kiedrowski G. A self-replicating hexadeoxynucleotide. Angew. Chem. Int. Ed. Engl., 25, 932-935 (1986).

[136] von Kiedrowski G. Minimal replicator theory I: Parabolic versus exponential growth. In Bioorganic Chemistry Frontiers, Volume 3, pp. 115-146 (Springer-Verlag, Berlin, Heidelberg, 1993).

[137] von Kiedrowski G., Wlotzka B., and Helbing J. Sequence dependence of templatedirected syntheses of hexadeoxynucleotide derivatives with 3'-5' pyrophosphate linkage. J. Angew. Chem. Int. Edn. engl., 28, 1235-1237 (1989).

[138] Vrba E.S. Levels of selection and sorting with special reference to the species level. In Oxford Surveys in Evolutionary Biology, P.H. Harvey and L. Partridge, eds., volume 6, pp. 114-115 (Oxford University Press, Oxford, 1989).

[139] Walde P., Wick R., Fresta M., Mangone A., and Luisi P.L. Autopoietic selfreproduction of fatty acid vesicles. J. Amer. Chem. Soc., 116, 11649-11654 (1994).

[140] Wetherill G.W. Formation of the Earth. Annu. Rev. Earth Planet. Sci., 18, 205-256 (1990).

[141] Wills P.R., Kauffman S.A., Stadler B.M., and Stadler P.F. Selection dynamics in autocatalytic systems: Templates replicating through binary ligation. Bull. Math. Biol., 60, 1073-1098 (1998).

[142] Wlotzka B. and McCaskill J.S. A molecular predator and its prey: Coupled isothermal amplification of nucleic acids. Chemistry \& Biology, 4, 25-33 (1997).

[143] Wright S. The roles of mutation, inbreeding, crossbreeding and selection in evolution. In Proceedings of the Sixth International Congress on Genetics, D.F. Jones, ed., volume 1, pp. 356-366 (Brooklyn Botanic Gardens, New York, 1932).

[144] Wright S. "surfaces" of selective value. Proc. Nat. Acad. Sci. USA, 58, 165-172 (1967).

[145] Yao S., Ghosh I., Zutshi R., and Chmielewski J. Selective amplification by autoand cross-catalysis in a replicating peptide system. Nature, 396, 447-450 (1998).

[146] Zintzaras E., Santos M., and Szathmáry E. "Living" under the challenge of information decay: The stochastic corrector model vs. hypercycles. J. Theor. Biol., 217, 167-181 (2002).

[147] Zubay G. and Mui T. Prebiotic synthesis of nucleotides. Origin Life Evol. Biosp., 31, 87-102 (2001). 